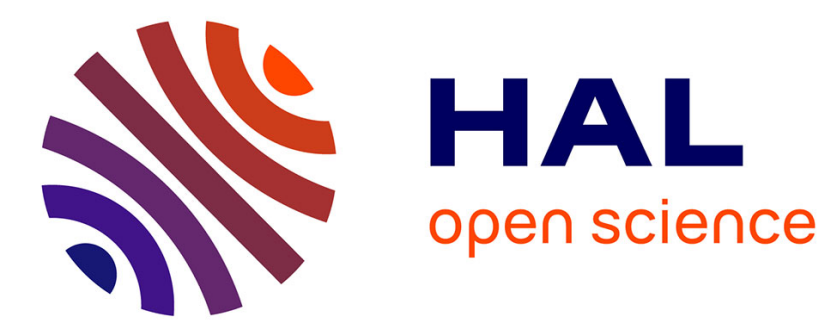

\title{
Ductile fracture of materials with randomly distributed voids
}

Clément Cadet, Jacques Besson, Sylvain Flouriot, Samuel Forest, Pierre

Kerfriden, Victor de Rancourt

\section{> To cite this version:}

Clément Cadet, Jacques Besson, Sylvain Flouriot, Samuel Forest, Pierre Kerfriden, et al.. Ductile fracture of materials with randomly distributed voids. International Journal of Fracture, 2021, 230, pp.193-223. 10.1007/s10704-021-00562-7 . hal-03372371

\section{HAL Id: hal-03372371 \\ https://hal.science/hal-03372371}

Submitted on 16 Oct 2021

HAL is a multi-disciplinary open access archive for the deposit and dissemination of scientific research documents, whether they are published or not. The documents may come from teaching and research institutions in France or abroad, or from public or private research centers.
L'archive ouverte pluridisciplinaire HAL, est destinée au dépôt et à la diffusion de documents scientifiques de niveau recherche, publiés ou non, émanant des établissements d'enseignement et de recherche français ou étrangers, des laboratoires publics ou privés. 


\title{
Ductile Fracture of Materials with Randomly Distributed Voids
}

\author{
Clément Cadet · Jacques Besson · Sylvain Flouriot · Samuel \\ Forest $^{\star}$ · Pierre Kerfriden · Victor de Rancourt
}

December 3rd, 2020

\begin{abstract}
A reliable determination of the onset of void coalescence is critical to the modelling of ductile fracture. Numerical models have been developed but rely mostly on analyses on single defect cells, thus underestimating the interaction between voids. This study aims to provide the first extensive analysis of the response of microstructures with random distributions of voids to various loading conditions and to characterize the dispersion of the results as a consequence of the randomness of the void distribution. Cells embedding a random distribution of identical spherical voids are generated within an elastoplastic matrix and subjected to a macroscopic loading with constant stress triaxiality and Lode parameter under periodic boundary conditions in finite element simulations. The failure of the cell is determined by a new indicator based on the loss of full rankedness on the average deformation gradient rate. It is shown that the strain field developing in random microstructures and the one in unit cells feature different dependencies on the Lode parameter $L$ owing to different failure modes. Depending on $L$, the cell may fail in extension (coalescence) or in shear. Moreover the random void populations lead to a significant dispersion of failure strain, which is present even in simulations with high numbers of voids.
\end{abstract}

Keywords Ductile Fracture $\cdot$ Void coalescence $\cdot$ Homogenization

* Corresponding author, E-mail: samuel.forest@mines-paristech.fr

C. Cadet · J. Besson · S. Forest · P. Kerfriden

MINES ParisTech, PSL University, MAT - Centre des Matériaux, CNRS UMR 7633, BP 87, 91003 Évry, France

E-mail: clement.cadet@mines-paristech.fr, jacques.besson@mines-paristech.fr, pierre.kerfriden@mines-paristech.fr

C. Cadet $\cdot$ S. Flouriot $\cdot$ V. de Rancourt

CEA Valduc, Is-sur-Tille, France

E-mail: sylvain.flouriot@cea.fr, victor.derancourt@cea.fr 


\section{Introduction}

2 Predicting the failure of a structural part subjected to monotonous loading requires a good understanding of the ductile fracture behavior of the material. However ductile fracture is a complex phenomenon involving a variety

4 of mechanisms, strongly dependent on the material and involving large strain at least on a local level (Besson, 5 2004). Voids are first nucleated within the material, especially near second phase inclusions. Depending on the 6 loading conditions, the voids may or may not grow. Finally the material fails when voids coalesce, either by 7 internal necking or by the nucleation of secondary voids (mostly for shear-dominated loading). Softening due to 8 void growth may also be sufficient to initiate failure without coalescence per se (Tekoğlu et al., 2015). Although 9 a large body of literature on ductile fracture has already been published, accurate failure prediction is still a 10 research problem, as evidenced by the Sandia fracture challenges (Boyce, Kramer, Fang, et al., 2014; Boyce, 11 Kramer, Bosiljevac, et al., 2016; Kramer et al., 2019): besides the difficulty of calibrating modellling parameters 12 from experimental data, predicting ductile failure requires to take into account many strongly nonlinear physical 13 processes.

Experimental studies have shown that the failure behavior strongly depends on the stress state to which the material is subjected. The effects of the stress triaxiality (ratio of the von Mises equivalent stress to the mean stress) and the Lode parameters (reflecting the third stress invariant) have been extensively investigated (for instance by Helbert et al. (1996), Bao and Wierzbicki (2004), Barsoum and Faleskog (2007), Gao et al. (2009), Dunand and Mohr (2011), Gilioli et al. (2013), Zhai et al. (2016), Xiao et al. (2018), and Zhang, Badreddine, et al. (2020)). Models representing ductile failure should therefore account for the effect of these two parameters.

Analytic and computational approaches at a micromechanical level have also been developed to investigate 21 the mechanisms of ductile fracture, to model ductile fracture and provide microscale-informed failure prediction. 22 Following Gurson's (1977) results from limit analysis, increasingly precise analytic models have been developed by explicitly representing approximate strain fields near voids in a plastic material. Besson (2010) provides a review of such models but more recent ones have been developed to represent void growth and coalescence either by necking or in shear (Benzerga and Leblond, 2014; Morin, Leblond, Benzerga, and Kondo, 2016; Torki, 2019;

so Faleskog, 2011; Zhu, Engelhardt, et al., 2018) or by distinguishing strain localization from coalescence (Wong 
and Guo, 2015; Guo and Wong, 2018; Zhu, Ben Bettaieb, et al., 2020). However these studies are mostly carried out on unit cells: the global behavior of the material is summarized by that of a meshed cell containing a single void. Even though this approach was proven useful to analyze fundamental mechanisms at the void level at a low computational cost, it oversimplifies the interaction between voids, whose influence increases with porosity, by assuming that voids are regularly distributed as a cubic lattice.

Some studies have investigated the interaction of voids in simplified configurations, involving only a couple of voids. For instance Bandstra and Koss (2008) considered three-voids clusters with rotational symmetry in an hexagonal volume element; Tvergaard (2016) and Tvergaard (2017) considered 2D clusters with three aligned pores, whereas Trejo Navas et al. (2018) systematically studied 3D three pore clusters. Khan and Bhasin (2017) investigated the interaction between two populations of voids, in the simplified context of a high symmetry periodic arrangement. However, in a real material, a large number of voids, with complex spatial distribution interact with each other. Shakoor et al. (2015) considered 2D microstructures with a random population of voids and showed that increased triaxiality accelerates coalescence. Shakoor et al. (2018) also provided a very fine description of the mechanisms of ductile fracture from nucleation up to coalescence, between randomly distributed voids. All these studies evidence the role of clusters but do not allow to compute coalescence properties depending on loading conditions, as a model of ductile fracture would require, because they investigate too few void configurations and loading cases.

Analytical approaches can take random void distributions into account. For instance, Leblond and Mottet (2008) developed a limit analysis model coupling coalescence and shear band formation initially for a periodic distribution, but proposed a method to extend it to the random case by considering all possible orientations of the shear bands. Moreover, works by Danas and Ponte Castañeda (2009) or Vincent et al. (2009) for instance, considered random void populations within the context of a nonlinear variational homogenization scheme: the porous medium was compared to a linear composite, whose stiffness is based on Ponte Castañeda and Willis's (1995) bounds, an effective method to represent a population of random elliptical voids. This variational technique was subsequently used by Danas and Ponte Castañeda (2012) to investigate the influence of stress triaxiality and Lode parameter. However, such analytical approaches should be compared to simulations to check the validity of their assumptions. For instance, Danas and Ponte Castañeda's (2012) predictions for the behavior at low triaxiality were found to be unrealistic by Hutchinson and Tvergaard's (2012) FEM simulations on unit cells with the same loading conditions.

Explicit simulations of random void distributions have been carried out in a limited number of works. Bilger, Auslender, Bornert, Michel, et al. (2005) and Bilger, Auslender, Bornert, Moulinec, et al. (2007) using 
62 Fast Fourier Transform then Fritzen, Forest, Böhlke, et al. (2012) with Finite Elements Analysis proposed a

computational homogenization method to determine an effective yield surface. Several microstructures consisting of a random void distribution embedded in a plastic matrix are simulated up to overall plastic yield for several loading conditions. The results are averaged over the several microstructures to determine an homogenized yield surface (represented for Fritzen, Forest, Böhlke, et al. (2012) by a GTN criterion). This approach was extended to a Green-type porous matrix (Fritzen, Forest, Kondo, et al., 2013), to multiple void populations of different size (Khdir et al., 2014) and to non-spherical voids (Khdir et al., 2015). However these studies were focused on yield surface and did not address coalescence. Recently, Hure (2021) did perform FFT simulations on cells with multiple voids up to coalescence, and illustrated the influence of the number of voids on the stress at coalescence. Yet this study was limited to the simple case of axisymmetric loading.

To the authors' knowledge, a description of coalescence for various loading conditions and at the level of a representative volume element with multiple voids, has not been done yet. We therefore propose here to extend the methodology of Fritzen, Forest, Böhlke, et al. (2012) and Hure (2021) to the study of coalescence under various stress states. We aim to assess the effect of the interaction between randomly distributed voids on the macroscopic failure response of a cell, depending of the stress state. The results should be compared to those of unit cells to identify how they differ from cells with multiple voids. Moreover the statistical dispersion in failure results linked to the random distribution should be quantified.

To this end, cells composed of a random population of identical spherical voids are generated and subjected to various loading conditions, characterized by constant stress triaxiality and Lode parameter levels, in finite element simulations with Z-set software (Besson and Foerch, 1998). We then propose a coalescence indicator based on the loss of full rankedness of the macroscopic deformation gradient rate. The identification of coalescence during the simulation allows to extract several quantities of interest at the onset of coalescence. Our main results show that the evolution of the onset of coalescence with respect to the Lode parameter is qualitatively different between random microstructures and unit cells. This difference is associated to a change of coalescence modes for random microstructures. Finally, dispersion of the results due to the randomness of the void distribution is studied.

Section 2 describes the methodology used to generate random microstructures and to prescribe the loading conditions within the FE simulation. In section 3 typical numerical simulation results are presented and an indicator is defined to identify failure. Section 4 applies the methodology of sections 2 and 3 to compare the response of random microstructures to that of a unit cell, both on the evolution of macroscopic (cell-level) quantities, and on plastic strain field patterns. The dispersion of the results is also investigated. Finally, we 
discuss in section 5 the simulation hypotheses chosen in this work, and verify to what extent the results can be generalized.

An intrinsic notation is used for tensors: vectors, as first order tensors, are represented as $\underline{v}=v_{i} \underline{e}_{i}$ and second order tensors as $\underset{\sim}{A}=A_{i j} \underline{e}_{i} \otimes \underline{e}_{j}$, where $\left(\underline{e}_{i}\right)$ is an orthonormal frame. The subscript 0 in the notation $A_{0}$ refers to the value of $A$ in the initial configuration at time $t=0$. The position of a material point initially at $\underline{x}_{0}$ evolves with time $t$ as $\underline{x}=\underline{\Phi}\left(\underline{x}_{0}, t\right)$; the deformation gradient is then defined as $\underset{\sim}{F}=\frac{\partial \Phi}{\partial \underline{x}_{0}}$. Quantities decorated with an overlying bar, such as $\bar{A}$, refer to the macroscopic counterpart (at the level of a cell) of a quantity $A$ defined locally. For instance $\bar{\sim}$ is the average deformation gradient (defined more precisely in section 2.3), and $\bar{J}=\operatorname{det}(\underset{\sim}{\bar{F}})$.

\section{Methodology}

\subsection{Generation of random microstructures and finite element meshing}

The methodology to create the elementary volumes follows that of Fritzen, Forest, Böhlke, et al. (2012). These cells consist of a cubic matrix containing a population of identical non overlapping spherical defects. As all the $N_{\text {defects }}$ spheres have the same radius $r$, the porosity of the cell (of size $L_{\text {cube }}$ and therefore of volume $V_{0}=L_{\text {cube }}^{3}$ ) is defined as:

$$
f=\frac{4 \pi}{3} N_{\text {defects }}\left(\frac{r}{L_{\text {cube }}}\right)^{3}
$$

The radius of the voids is fully determined once the porosity and the number of voids are chosen. The initial porosity was chosen as $f_{0}=6 \%$, to be compared to the range of porosity levels $f_{0} \in[0.1 \%, 30 \%]$ considered by Fritzen, Forest, Böhlke, et al. (2012). However unit cell analyses frequently study lower porosities, with $f_{0} \sim 0.1 \%$ (Wong and Guo, 2015; Vishwakarma and Keralavarma, 2019; Guo and Wong, 2018). For low porosity values, interactions between defects can indeed be neglected (Koplik and Needleman, 1988), at least for the growth phase. Fritzen, Forest, Böhlke, et al., 2012 showed for instance that unit cells and random microstructures with sufficiently low prosity levels have a similar growth behavior, which can be represented by a GTN criterion Nonetheless, high porosity levels of $6 \%$ are possible in sintered materials (Becker, 1987), nodular cast iron (Zhang, Bai, et al., 1999), irradiated stainless steel (Cawthorne and Fulton, 1967). Moreover, overall porosity of $0.5 \%$ to $2 \%$ can be found in weld joints (Li et al., 2003; Sarre, 2018; Lacourt, 2019), but porosity values defined at a smaller scale, near void clusters, can be higher. A high initial porosity level can also provide insight for coalescence at lower initial porosity levels. Coalescence starts after a sufficient phase of growth so that voids 
begin interacting with each other and can no longer be considered isolated, which means that the porosity is no longer negligible. Notwithstanding the change of void shape which will play a significant role, starting at high porosity is equivalent, to some extent, to considering the end of a simulation at lower porosity.

The position of the defects is chosen according to a Poisson sphere process (Matern, 1986). As the target porosity is significantly lower than the jamming porosity levels that characterize such processes (around $38 \%$ according to Gamito and Maddock (2009)), a dart-throwing method is sufficient for the sampling. The position of the center of a sphere is chosen according to a uniform distribution on the cube. If the distance between the resulting spheres and the already built defects is larger than $10 \%$ of the radius of a sphere, the new sphere is included in the list of defects. Otherwise it is rejected and a new possible center is chosen randomly. Introducing a repulsion distance between the defects allows a better mesh quality. During the FEM simulations, periodic boundary conditions are applied (see section 2.3). Therefore a periodic microstructure and in turn a periodic mesh should be used. In order to ensure the periodicity of the population of defects, each time a new defect intersects a side of the cube, it is copied on the other side (thus there are four copies if an edge is intersected, and eight if the defect contains a vertex of the cube). All of these copies are taken into account to determine intersections between defects. Fritzen, Forest, Böhlke, et al. (2012) verified several statistical properties of the representativeness of this process.

The cell with the preceding defect population is meshed with NETGEN software (Schöberl, 1997). This tool first meshes surfaces, then volumes, and generates a non structured tetrahedral mesh. Periodicity of the mesh is imposed so that opposite sides of the cube have identical surface meshes. A maximum element size of $h_{\text {cell }}=r$ is imposed globally on the cell, but on the surface meshes of the defects the maximum element size is reduced to $h_{\text {void }}=r / 5$. The mesh is thus refined on the part of the surface mesh corresponding to the surface of voids. Finally, tetrahedral second-order 10-nodes elements with reduced integration are used to limit volume locking (due to large strain plasticity) in the FEA simulations. An example of the meshing of a microstructure with 27 cells is shown in figure 1a.

As cells are cubic, they define a canonical orthonormal frame $\left(O, \underline{e}_{1}, \underline{e}_{2}, \underline{e}_{3}\right)$ where $O$ is a vertex of the cube and the unit vectors $\underline{e}_{1}, \underline{e}_{2}, \underline{e}_{3}$ are parallel to edges of the cube (in the initial configuration). All tensor components will be expressed in this frame.

Although a diversity of microstructures were used in this study, several are repeatedly referred to in this article; they are shown in figure 1 . The microstructures $R 1$ and $R 2$ are two random microstructures with 27-voids of radius $r=0.08 L_{\text {cube }, R 1}$. The one-pore cell unit is defined as a cubic matrix of size $L_{\text {cube }, \text { unit }}=L_{\text {cube }, R 1} / 3$ containing a unique defect of radius $r=0.08 L_{c u b e, R 1}$ (same radius as before, and thus same volume fraction). It 
is meshed with the same procedure and same parameters as the larger cells with a random population. Finally the microstructure lattice consists in $3 \times 3 \times 3$ defects arranged on a cubic lattice; it is meshed in the same way as the random microstructures, so the mesh is not the assemblage of 27 small identical meshes of the unit cells.

2.2 Material behavior law at finite strain

Finite element simulations are carried out using Zset software (Besson and Foerch, 1998; www.zset-software.com 2020). As the matrix can undergo large deformation before coalescence, the simulations are performed in a finite strain framework. A local objective frame approach is adopted to formulate the constitutive law of the matrix (Besson, Cailletaud, et al., 2009). The strain rate $\underset{\sim}{D}$ and Cauchy stress $\underset{\sim}{\sigma}$ tensors are convected in a corotational frame:

$$
\begin{aligned}
& \underset{\sim}{\dot{e}}=\underset{\sim}{Q^{T}} \underset{\sim}{\underset{\sim}{Q}} \\
& \underset{\sim}{s}=J{\underset{\sim}{Q}}^{T} \underset{\sim}{\sigma} \underset{\sim}{Q}
\end{aligned}
$$

where $\underset{\sim}{Q}$ is a rotation matrix verifying $-{\underset{\sim}{Q}}^{T} \underset{\sim}{\dot{Q}}={\underset{\sim}{\dot{Q}}}^{T} \underset{\sim}{\underset{\sim}{Q}}=\underset{\sim}{W}\left(\underset{\sim}{W}=\operatorname{skew}\left(\underset{\sim}{\dot{F}} F^{-1}\right)\right.$ is the material spin tensor $)$. This choice of corotational frame is equivalent to using the Jaumann derivative of the stress in the hypo-elasticity law.

The constitutive law is then defined by a classical additive decomposition of convected strain rates in a isotropic elastic part and a plastic part. Isotropy and time-independent perfect plasticity (absence of hardening) with a von Mises yield criterion are assumed for the matrix material:

$$
\begin{aligned}
& \stackrel{\dot{e}}{=} \dot{e}_{e}+\dot{e}_{p} \\
& e_{e}=\frac{1+v}{E} \underset{\sim}{s}-\frac{v}{E}(\operatorname{tr} \underset{\sim}{s}) \underset{\sim}{1} \\
& s_{v m}=\sqrt{\frac{3}{2} s^{d e v}: s_{\sim}^{d e v}} \\
& f(\underset{\sim}{s})=s_{v m}-R_{0} \leq 0 \\
& \dot{\sim}_{p}=\dot{p} \frac{\partial f}{\partial \underset{\sim}{s}}
\end{aligned}
$$

with ${\underset{\sim}{d e v}}^{d e v}$ the deviatoric part of the rotated Cauchy stress tensor $\underset{\sim}{s}, s_{v m}$ the equivalent von Mises stress and $\dot{p}=\sqrt{\frac{2}{3} \dot{e}_{p}: \dot{e}_{p}}$ playing the role of the plastic multiplier. The Young modulus, the Poisson ratio and the yield strength are respectively chosen as $E=200 \mathrm{GPa}, v=0.3$ and $R_{0}=500 \mathrm{MPa}$, hence $R_{0} / E=0.0025$. The 

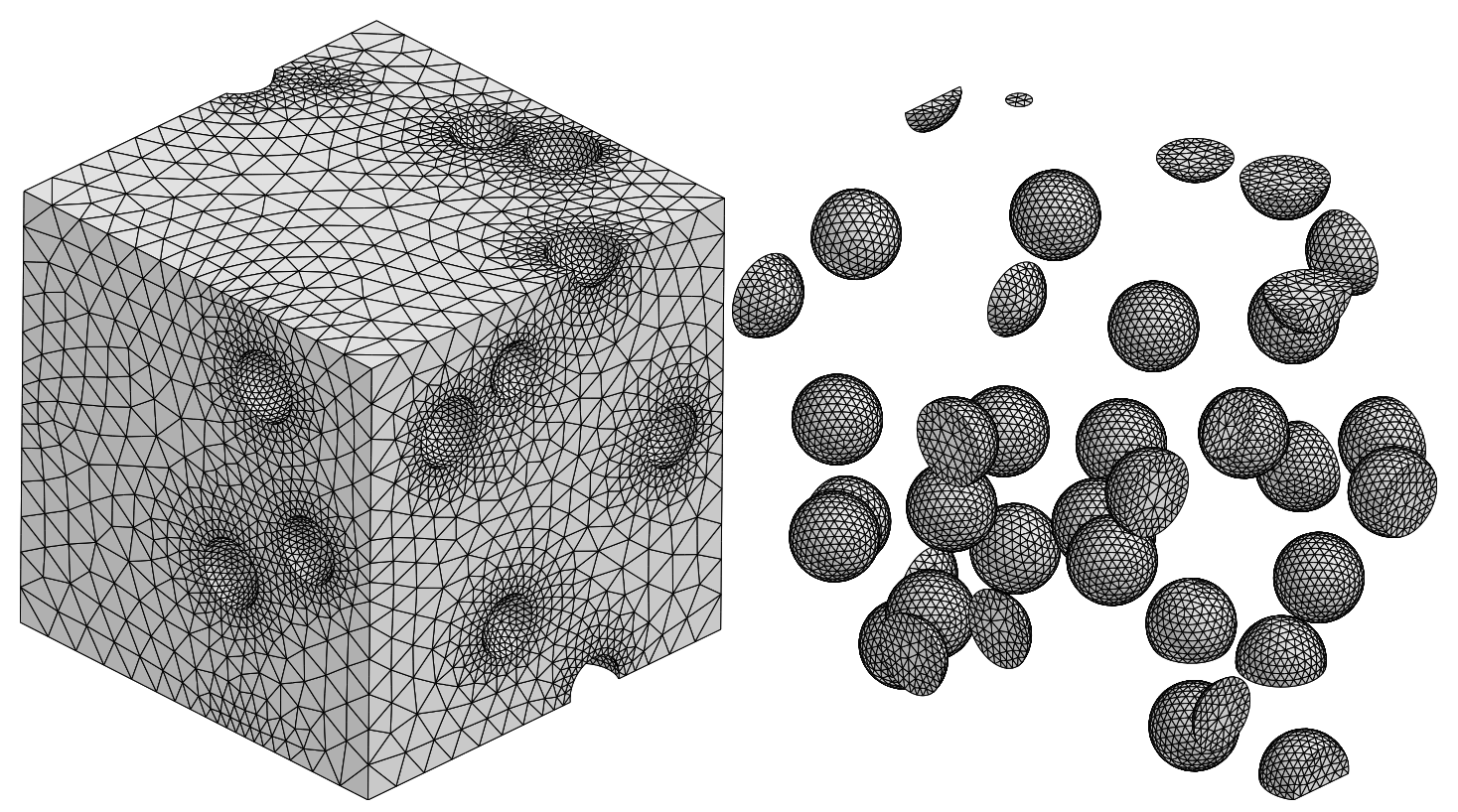

(a) Random microstructure $R 1$ with 27 voids (FE mesh with 255628 nodes).
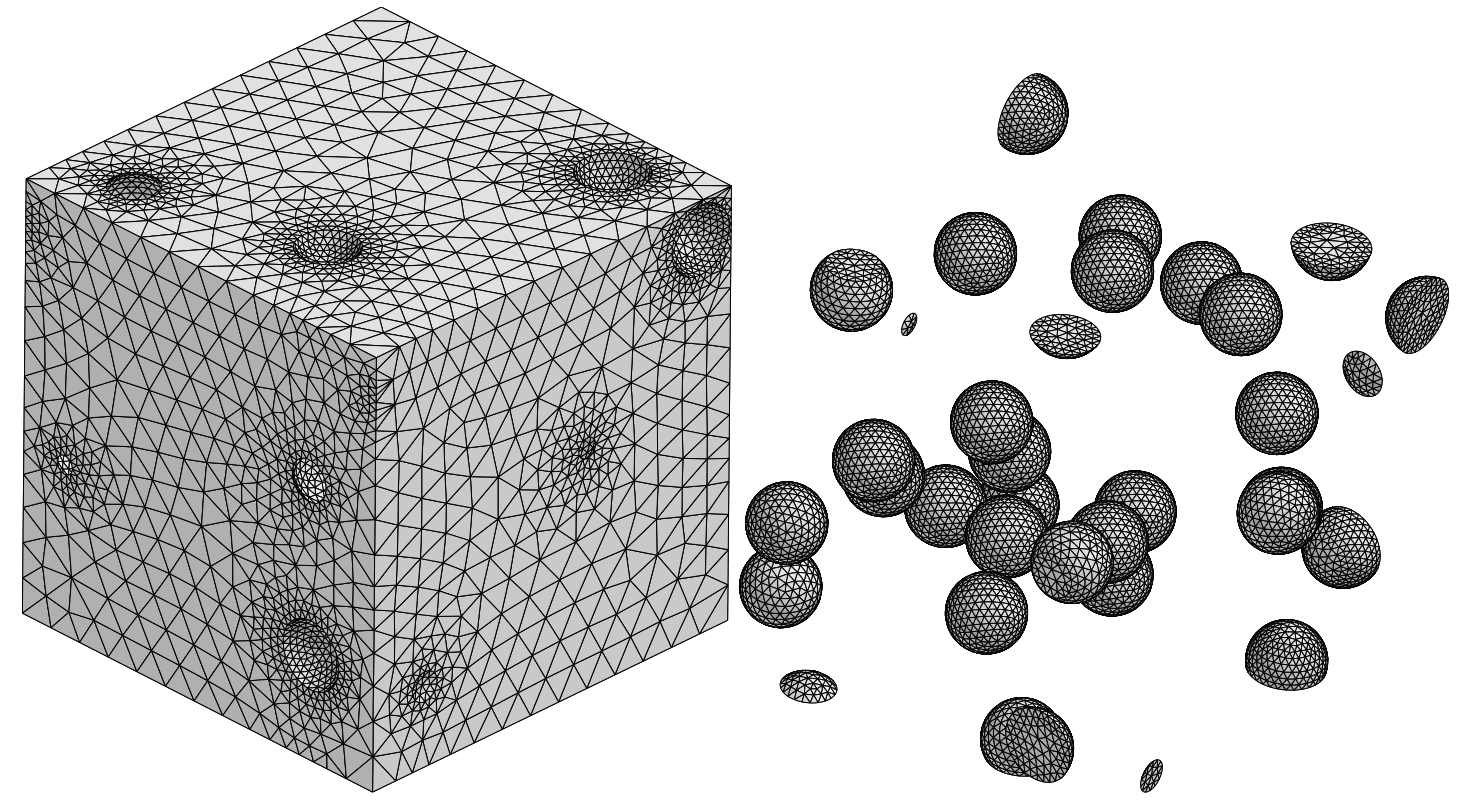

(b) Random microstructure $R 2$ with 27 voids (FE mesh with 176982 nodes).

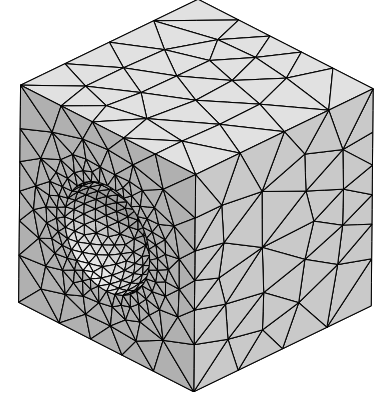

(c) Unit cell with one void.

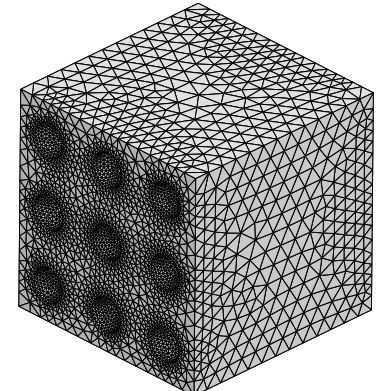

(d) Microstructure lattice with 27 voids on a cubic lattice.

Fig. 1: Meshes of some microstructures repeatedly used in the study. 
cumulative plastic strain is defined as:

$$
p=\int_{0}^{t} \dot{p} d t
$$

where $t$ is actually a fictitious time in rate-independent plasticity, acting as an increasing loading parameter.

During the finite element analysis, this constitutive law is integrated at each quadrature point of the finite element mesh by an implicit Euler method, then the global static equilibrium is solved in total Lagrangian formulation by a Newton-Raphson scheme with a consistent tangent matrix.

\subsection{Boundary and loading conditions}

The boundary and loading conditions follow that of Ling et al. (2016). Periodic boundary conditions are applied on the sides of the cube (Besson, Cailletaud, et al., 2009). The displacement field $\underline{u}$ should therefore have the form:

$$
\underline{u}=\left(\underline{\sim}_{\sim}-\underline{\sim}^{1}\right) \cdot \underline{x}_{0}+\underline{v}\left(\underline{x}_{0}\right)
$$

with $\underset{\sim}{\bar{F}}$ the average deformation gradient, and $\underline{v}$ a displacement fluctuation field, periodic and with zero average over the cell. The periodicity of $\underline{v}$ and the anti-periodicity of traction vectors mean that:

$$
\begin{gathered}
\underline{v}\left(\underline{x}_{0}^{+}\right)=\underline{v}\left(\underline{x}_{0}^{-}\right) \\
\underline{\sim} \cdot \underline{n}\left(\underline{x}_{0}^{+}\right)=-\underline{\sim}_{0} \cdot \underline{n}\left(\underline{x}_{0}^{-}\right)
\end{gathered}
$$

if $\underline{x}_{0}^{+}$and $\underline{x}_{0}^{-}$represent two homologous points on opposite sides of the periodic mesh and $\underline{n}\left(\underline{x}_{0}\right)$ represent the outward-pointing normal to the mesh boundary at $\underline{x}_{0}$. In this formulation, the degrees of freedom are the three components of the displacement fluctuation field for each node of the mesh and the nine components of $\bar{\sim}$ (or rather of $\underset{\sim}{E}=\underset{\sim}{\bar{F}}-\underset{\sim}{1})$.

The macroscopic Boussinesq (or first Piola-Kirchhoff) and Cauchy stress tensors are defined by:

$$
\begin{aligned}
& \underset{\sim}{S}=\frac{1}{V_{0}} \int_{V_{0}} \underset{\sim}{S} \mathrm{~d} V_{0}=\frac{1-f_{0}}{V_{0}} \int_{V_{0}^{\text {matrix }}} \underset{\sim}{S} \mathrm{~d} V_{0} \\
& \bar{\sim}=\frac{1}{\bar{J}} \underset{\sim}{\bar{S}} . \bar{\sim}^{T}
\end{aligned}
$$

where $\bar{J}=\operatorname{det}(\underset{\sim}{\bar{F}})$ and $V_{0}$ is the volume of the cell (matrix and defects) in the initial configuration. The integration on $V_{0}$ implicitly considers that stress is well-defined and identically zero in the voids. 
The simulations are carried out at constant (macroscopic) stress triaxiality and Lode parameter. These quantities are here defined as:

$$
\begin{aligned}
T & =\frac{\operatorname{tr} \underset{\widetilde{\sigma}}{\bar{\sigma}}}{3 \bar{\sigma}_{v m}} \\
L & =\frac{2 \bar{\sigma}_{2}-\bar{\sigma}_{1}-\bar{\sigma}_{3}}{\bar{\sigma}_{1}-\bar{\sigma}_{3}}
\end{aligned}
$$

where $\bar{\sigma}_{e q}$ is the von Mises equivalent stress calculated from $\underset{\sim}{\bar{\sigma}}$ and $\bar{\sigma}_{1} \geq \bar{\sigma}_{2} \geq \bar{\sigma}_{3}$ (with $\bar{\sigma}_{1}>\bar{\sigma}_{3}$ ) are the three eigenvalues of $\sigma . L=-1, L=0$ and $L=1$ respectively correspond to states of generalized tension, shear and compression. An alternative definition of a Lode parameter with $L=1$ for tension and $L=-1$ for compression can also be found in literature (e.g. Barsoum and Faleskog (2011) and Wong and Guo (2015))

To ensure that $T$ and $L$ remain constant during the simulation, a special macroscopic spring element was developed by Ling et al. (2016). It acts on the $E_{i j}$ degrees of freedom, and its reaction forces are calculated so that $\underset{\sim}{\bar{\sigma}}$ keeps the following diagonal form throughout the simulation:

$$
\bar{\sigma}=\left[\begin{array}{ccc}
\bar{\sigma}_{1} & 0 & 0 \\
0 & \bar{\sigma}_{2} & 0 \\
0 & 0 & \bar{\sigma}_{3}
\end{array}\right]=\bar{\sigma}_{11}\left[\begin{array}{ccc}
1 & 0 & 0 \\
0 & \eta_{2} & 0 \\
0 & 0 & \eta_{3}
\end{array}\right]
$$

where $\eta_{2}=\bar{\sigma}_{2} / \bar{\sigma}_{1}$ and $\eta_{3}=\bar{\sigma}_{3} / \bar{\sigma}_{1}$ are prescribed constants which define the stress state. Therefore the eigenvectors of $\underset{\sim}{\bar{\sigma}}$ are collinear to the three axes of the cube.

Unlike Barsoum and Faleskog (2011), Dunand and Mohr (2014), Wong and Guo (2015), and Zhu, Engelhardt, et al. (2018) but like Zhu, Ben Bettaieb, et al. (2020) and Guo, Ling, et al. (2020), we chose not to consider the effect of a shear stress component (for instance $\bar{\sigma}_{12}$ ) for computational cost reasons. However the cubic cell exhibits cubic symmetry and has an anisotropic behavior. The additional stress component could allow different loading orientations with identical $T$ and $L$ values. The consequences of this choice will be discussed in section 5.1.

To prevent degeneracy of solutions due to rigid body motion, a global translation and a global rotation of the cube should be fixed. The translation is taken care of by fixing a vertex of the cube. For the rotation, a possible method is to impose three additional constraints on the average deformation gradient $\underset{\sim}{F}$. For instance $\underset{\sim}{F}$ can be supposed symmetric, as done by Ling et al. (2016):

$$
\bar{F}_{12}=\bar{F}_{21} \quad \bar{F}_{23}=\bar{F}_{32} \quad \bar{F}_{13}=\bar{F}_{31}
$$


Another method is presented and discussed in appendix B.2.

With the aforementioned conditions, the simulation can be strain-controlled by specifying only the average strain along the first axis $E_{11}=\bar{F}_{11}-1$. We impose $E_{11}=\dot{\varepsilon} t$, with $\dot{\varepsilon}$ an arbitrary strain rate (the value can be arbitrarily chosen, as the plasticity is time-independent). At the beginning of the simulation $t=0$, the cell is undeformed, and $\underset{\sim}{\bar{F}}=1$.

\section{Description of a coalescence indicator}

\subsection{Typical results of a computation}

With the method described in the previous subsection, simulations can be carried out for several loading conditions. In this study, we are interested in the evolution of several quantities at failure. However defining ductile failure and detecting it during the simulation is not straightforward. To illustrate this issue, some enlightening simulation results will be described first.

Three simulations on the microstructure $R l$ were carried out in generalized tension $(L=-1)$ at three triaxiality levels $T=0.8, T=1$ and $T=1.4$. The figure 2a compares the macroscopic Cauchy and Boussinesq stress components along the main loading axis (the marker on the curves corresponds to the indicator described later in section 3.3). The three loading conditions lead to a similar evolution of stress. The stress maximum is reached shortly after the beginning of the computation (for $E_{11}<0.01$ ) then the stress decreases monotonously and almost linearly. However, at a critical strain that depends on the loading condition, the decrease in stress suddenly accelerates and the unit cell quickly loses all its load-bearing capacity (at approximately $E_{11}=0.5$, $0.35,0.18$ for $T=0.8,1,1.4$ respectively). This event can be thought as the failure of the cell. Moreover, at the same strain as the onset of stress drop, the transverse strain $E_{22}$ stabilizes (fig. 2b). This macroscopic failure is also related to the behavior at a more microscopic level. Figure 2 shows the cumulative strain field $p$ shortly after this failure, for $T=1$ : plastic strain is concentrated in a band, mostly parallel to a side of the cube (and perpendicular to the main loading axis) but its exact shape fits closely the distribution of voids.

Although the stress decrease acceleration is clearly visible on the stress-strain plots in generalized tension, it is difficult to define its exact location so as to determine the precise failure onset and compute relevant physical quantities at this instant. Moreover the stress decrease does not generalize to shear-dominated loading conditions.

Therefore a more precise failure indicator remains to be determined. 


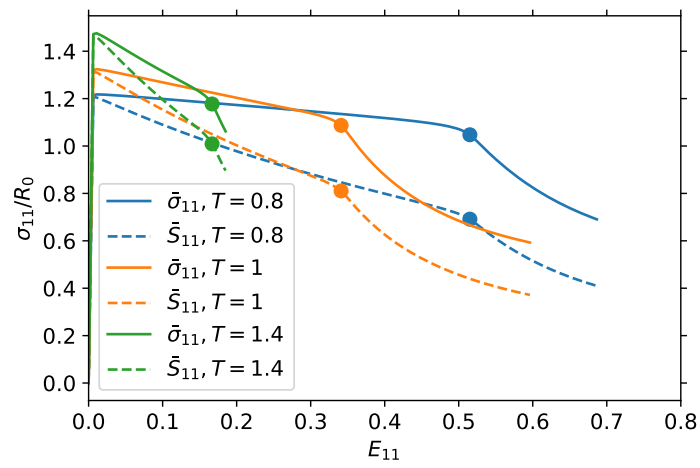

(a) Evolution of stress for three triaxiality levels at $L=-1$.
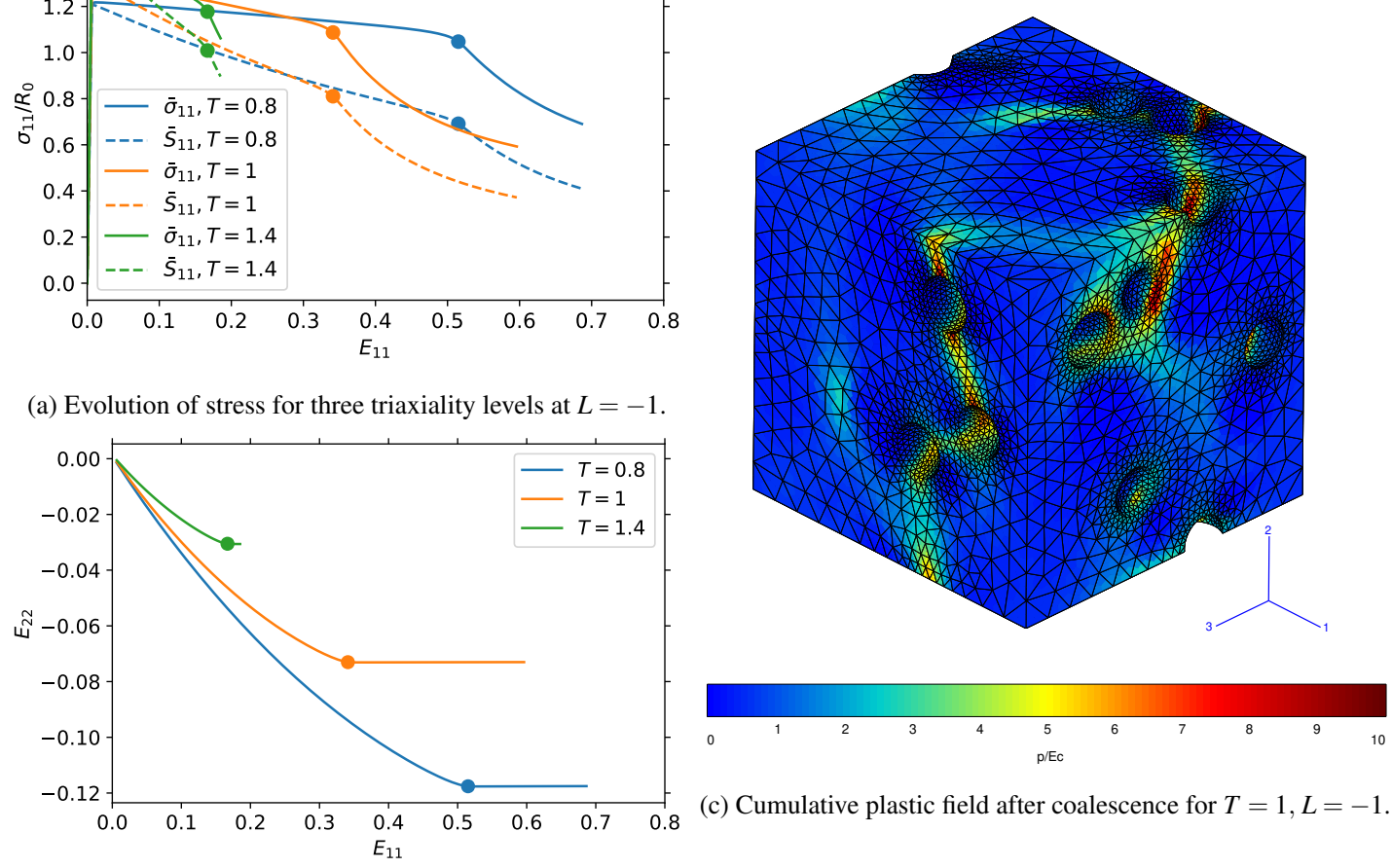

(c) Cumulative plastic field after coalescence for $T=1, L=-1$.

(b) Transverse strain for three triaxiality levels at $L=-1$.

Fig. 2: Typical computation results for the $R 1$ microstructure.

3.2 Available failure indicators in the literature

Several criteria for ductile failure in a unit cell have been developed, and are reviewed for instance by Zhu, Ben Bettaieb, et al. (2020). The earliest approaches were purely geometrical: Brown and Embury's (1973) criterion determines when strain bands are oriented at $45^{\circ}$ relative to the main loading axis, whereas McClintock (1968) and Tvergaard and Needleman (1984) (who modified Gurson's (1977) model) consider a critical porosity. Following Needleman and Tvergaard's (1992) work, a class of criteria determines the instant when strain is no more homogeneous and concentrates in the ligaments between voids. These criteria compare the norm of the strain rate in a localization band and its value outside the band (or the average value throughout the unit cell): if the ratio is higher than an arbitrarily chosen value, failure is said to have been reached. Such criteria are used for example by Barsoum and Faleskog (2007) or Dunand and Mohr (2014). Similarly, Luo and Gao (2018) and Vishwakarma and Keralavarma (2019) consider unit cells composed of several layers and force strain localization to happen in the central one (because the external layers contain smaller voids or no 
voids at all): failure can then be monitored by comparing the behavior of the layers. Another class of criteria determines when a maximum stress or force is reached. Such criteria can be derived by limit analyses, for instance Thomason (1985), Benzerga and Leblond (2014) or Morin, Leblond, Benzerga, and Kondo (2016). Guo and Wong (2018) interpreted the maximum of an effective force in terms of Rice's (1976) criterion on strain localization. Another approach, adopted by Koplik and Needleman (1988) and used for example by Ling et al. (2016) defines coalescence as the transition to a specific strain state: in coalescence, ligaments are in a state of uniaxial straining (whereas the rest of the cell is rigid and hardly deforms). Coalescence could also be interpreted in terms of plastic and elastic energy, as done by Wong and Guo (2015). A last approach was proposed by Zhu, Ben Bettaieb, et al. (2020) and involves computing the macroscopic acoustic tensor in order to directly apply Rice's (1976) criterion on strain localization.

However, as pointed for instance by Tekoğlu et al. (2015), Guo and Wong (2018) or Zhu, Ben Bettaieb, et al. (2020), the above criteria actually described two different physical processes: strain localization and coalescence During strain localization, strain concentrates in narrow bands, which can be interpreted as a loss of ellipticity, according to Rice's (1976) analysis. As stated previously, Guo and Wong (2018) establish a link between strain localization (through Rice's criterion) and maximum force criteria. Nonetheless, the more direct application of Rice's criterion by Zhu, Ben Bettaieb, et al. (2020) detects localization significantly later than Guo and Wong's (2018) interpretation. On the other hand, coalescence represents the fusion of several voids into a unique larger void during ductile failure. However the material model described in this article contains no ingredient to represent explicitly this process of coalescing voids. The state of coalescence can be deduced nevertheless from the FEM results: at some point in the loading, the cell stops thinning and the plastic flow inside becomes macroscopically uniaxial according to Koplik and Needleman (1988).

As the figure 2a shows, the cell's failure, defined by the sudden acceleration of the stress decrease, is incorrectly predicted by the instant of maximum force applied on the cell (with our choice of periodic boundary conditions, this force is here represented by $S_{11}$ ). Due to the absence of hardening, the maximum of $S_{11}$ happens at the beginning in the simulation, much earlier than the sudden stress drop. On the other hand, this stress drop occurs simultaneously with the stabilization of deformation in the 2-direction transverse to the main loading 1-axis, and can thus be associated with coalescence: the stabilization of the average transverse deformation indicates that the macroscopic strain becomes purely uniaxial. Coalescence thus seems an accurate failure indicator in this situation. According to Zhu, Ben Bettaieb, et al. (2020), an ellipticity loss approach based on the computation of the macroscopic acoustic tensor could also give sensible values of failure strains. This criterion was found to predict slightly earlier failure than a coalescence indicator. However Morin, Blystad Dæhli, et al. 
(2019) tried to apply coalescence and strain localization approaches to match experimental results; both gave acceptable results, with slightly better results for coalescence. Therefore we will focus on the coalescence approach.

3.3 Failure indicator based on the loss of full rankedness of $\underset{\sim}{\dot{\vec{F}}}$

The criterion of the stabilization of transverse displacement, as used by Ling et al. (2016), suffers from two main drawbacks. In a random population of voids, strain localization bands might not be parallel to a face of the cube, so monitoring $E_{22}$ with respect to $E_{11}$ might not detect coalescence. Moreover, this criterion is limited to the detection of coalescence by internal necking where voids coalesce in the plane orthogonal to the main loading axis. However, for shear dominated loading conditions (when the Lode parameter is close to zero), coalescence is known to occur in shear bands (Barsoum and Faleskog, 2007; Barsoum and Faleskog, 2011). We generalize here the stabilization of transverse deformation by noting that for both internal necking and shear, deformation gradient has a specific form during coalescence. After coalescence, there exist orthogonal unit vectors $\underline{e}$ and $\underline{e}^{\prime}$ such that $\underset{\sim}{\bar{F}}=\underset{\sim}{1}+\dot{\varepsilon} t \underline{e} \otimes \underline{e}$ for uniaxial straining and $\underset{\sim}{\bar{F}}=\underset{\sim}{1}+\dot{\varepsilon} t\left(\underline{e} \otimes \underline{e}^{\prime}+\underline{e}^{\prime} \otimes \underline{e}\right)$ for pure shear. In both cases, $\operatorname{det}(\dot{\overrightarrow{\bar{F}}})=0$. Therefore, as coalescence takes place, $\operatorname{det}(\underset{\sim}{\dot{\bar{F}}})$ should vanish.

This behavior of $\operatorname{det}(\underset{\sim}{\dot{F}})$ should be compared to the homogeneous plastic deformation case (which is an approximation, since strain may be concentrated in some ligaments). Let us then consider the function:

$$
\delta(t)=\dot{\varepsilon}^{-3}(1+\dot{\varepsilon} t)^{3} \operatorname{det}(\underset{\sim}{\dot{\bar{F}}})
$$

which compares the evolution of $\operatorname{det}(\underset{\sim}{\dot{\bar{F}}})$ to its theoretical evolution in the case of homogeneous compressible plastic flow. A derivation of the expression of $\delta$ and an example can be found in appendix A. Therefore, if $\delta(t) \rightarrow 0, \operatorname{det}(\underset{\sim}{\dot{\bar{F}}})$ decreases faster than expected by homogeneous plastic flow, and localization can be considered to have taken place.

The onset of failure can then be defined as the first instant $t_{c}$ such that:

$$
\delta\left(t_{c}\right) \leq \min \left(A \max _{t<t_{c}} \delta(t), B\right)
$$
where $A=0.05$ is a threshold comparing the maximal and current values of $\delta$ and $B=0.005$ is an absolute threshold. As shown by the $\alpha_{2}$ factor in equation (33), $\delta$ keeps smaller values for simulations with $L$ close to zero. In these cases, the relative threshold (depending on $A$ ) was found to be inappropriate due to numerical 
errors, and an absolute threshold $B$ (consistent with the value of $A$ ) was implemented; it is only needed for loading conditions with $|L|<0.3$. A sensitivity analysis with respect to the empirically chosen values $A$ and $B$ is carried out in appendix A and shows that the results which will be presented in section 4 are not strongly influenced by the values chosen for $A$ and $B$. The indicator is therefore robust with respect to the choice of these parameters.

As this criterion using the $\delta$ function relies only on macroscopic quantities (at cell-level), it is easy to compute and does not make any assumption on the position and orientation of the possible strain localizations. Moreover it can be used as a landmark in order to stop the simulations shortly after failure in order to spare computation time. However, the indicator detects a loss of full rank of the deformation gradient rate, and is therefore not adapted to loading conditions where the deformation gradient rate is intrinsically of rank 1 or 2. This is especially the case for $L=0$ for which the material is initially in shear, so that the indicator is activated in the elastic regime and predicts an early failure. This is acceptable for a perfectly plastic von Mises matrix, but leads to an underestimation of the strain at failure for materials whose hardening behavior delays coalescence. Moreover it is not able to represent a third and rarer form of coalescence known as necklace coalescence. This form was studied by Gologanu et al. (2001) for a cylindrical unit cell with an axisymmetric loading corresponding to our $L=1$ situation. The coalescence between voids takes place along the cylinder axis, which corresponds in our situation to the third and least stressed axis. Necklace coalescence is not associated to a loss of full rank, so the $\delta$ indicator cannot be activated. However for the loading conditions involving overall stress triaxiality considered in this work, the proposed indicator has been found to be relevant in all cases.

The failure onset $t_{c}$ can be determined with this method for the different loading conditions, and allows to define several quantities at the onset of coalescence: deformation at coalescence $E_{c}=E_{11}\left(t_{c}\right)$, stress at coalescence $\sigma_{c}=\bar{\sigma}_{11}\left(t_{c}\right)$ and porosity at coalescence $f_{c}=f\left(t_{c}\right)$. In the following, the evolution of those quantities and their dispersion due to the randomness of microstructures will be studied with respect to $T$ and $L$ parameters.

\section{Results}

4.1 Response of a microstructure subjected to proportional loading with different stress triaxiality and Lode parameter values

The random microstructures constructed in section 2.1 have two main differences in comparison to standard unit cells: they contain several voids and these voids are located irregularly within the cell. In this section, the 
effect of these differences on the behavior of cells is investigated. Several microstructures are considered and subjected to various loading conditions (defined by $T$ and $L$ ). Their failure behavior (evolution of $E_{c}, f_{c}$ and $\sigma_{c}$ with respect to $T$ and $L$ ) are then compared. The four microstructures shown on figure 1 are analysed: two random 27 -void cells $R 1$ and $R 2$, a unit cell, and a 27 -void cell, lattice, where the voids are distributed following a $3 \times 3 \times 3$ cubic cell. The four microstructures have the same porosity $6 \%$, the same pore size and are meshed with identical mesh size requirements in order to limit the influence of mesh convergence on the comparison (the mesh of the unit cell is thus composed of significantly fewer elements than the other three microstructures). Mesh size will be further discussed in section B.1. The lattice cell allows to separate effects due to the presence of several voids in the cell from those due to the irregular void distribution.

Although the failure behavior on the whole $T-L$ space should be explored, it is instructive to first consider constant triaxiality or constant Lode parameter slices of this space. Let us concentrate first on axisymmetric loading cases characterised by fixed $L=-1$, as in Ling et al. (2016). We focus on the zone of intermediate triaxiality $T \in[0.7,2.0]$, as usual in unit cell studies (Guo and Wong, 2018; Vishwakarma and Keralavarma, 2019). We did not study the very low triaxiality regime $T<0.4$ where the phenomenon of void collapse takes place (Bao and Wierzbicki, 2004; Liu et al., 2016). Triaxiality levels $T \in[0.4,0.7]$ were not studied so as to limit the duration of simulations: coalescence generally happens with the same mechanisms as for $T>0.7$, but at significantly higher strain values.

The evolution of $E_{c}, f_{c}$, and $\sigma_{c}$ with respect to $T$ are shown on the left side of figure 3 . The four microstructures display globally similar responses: $E_{c}$ decreases monotonously with increasing $\mathrm{T}$ while $\sigma_{c}$ increases linearly with $T . f_{c}$ behaves similarly to $E_{c}$, except for the microstructure $R 2: f_{c}$ is still a mainly decreasing function of $T$ but a local maximum is found at $T=1.2$. Note that the evolution of $E_{c}$ with respect to $T$ is smoother and less noisy than that of $\sigma_{c}$ (for $T=0.8$, the stress value for the lattice cell is for instance particularly low, when compared to the values at $T=0.6$ or $T=1.0$ ). A possible explanation is that, unlike $E_{11}$ which is linearly increasing with time, $\bar{\sigma}_{11}$ and $f$ vary rapidly around the instant of coalescence: $\bar{\sigma}_{11}$ decreases sharply around the coalescence (as evidenced by figure 15). Note also that for R2, the porosity at coalescence for $T=1.2$ is larger than for $T=1.1$, in contradiction to the overall evolution. Coalescence is detected at approximately the same strain in these two conditions, but as the porosity grows faster with increasing triaxiality, the porosity at coalescence is larger for $T=1.2$ than for $T=1.1$. This slight deviation from the overall evolution with $T$ seems due to the randomness of the void population.

The evolution of strain at coalescence $E_{c}$ was plotted in a logarithmic scale, so as to illustrate the exponential decrease for each microstructure. According to Rice and Tracey's (1969) results, a spherical void typical growth 

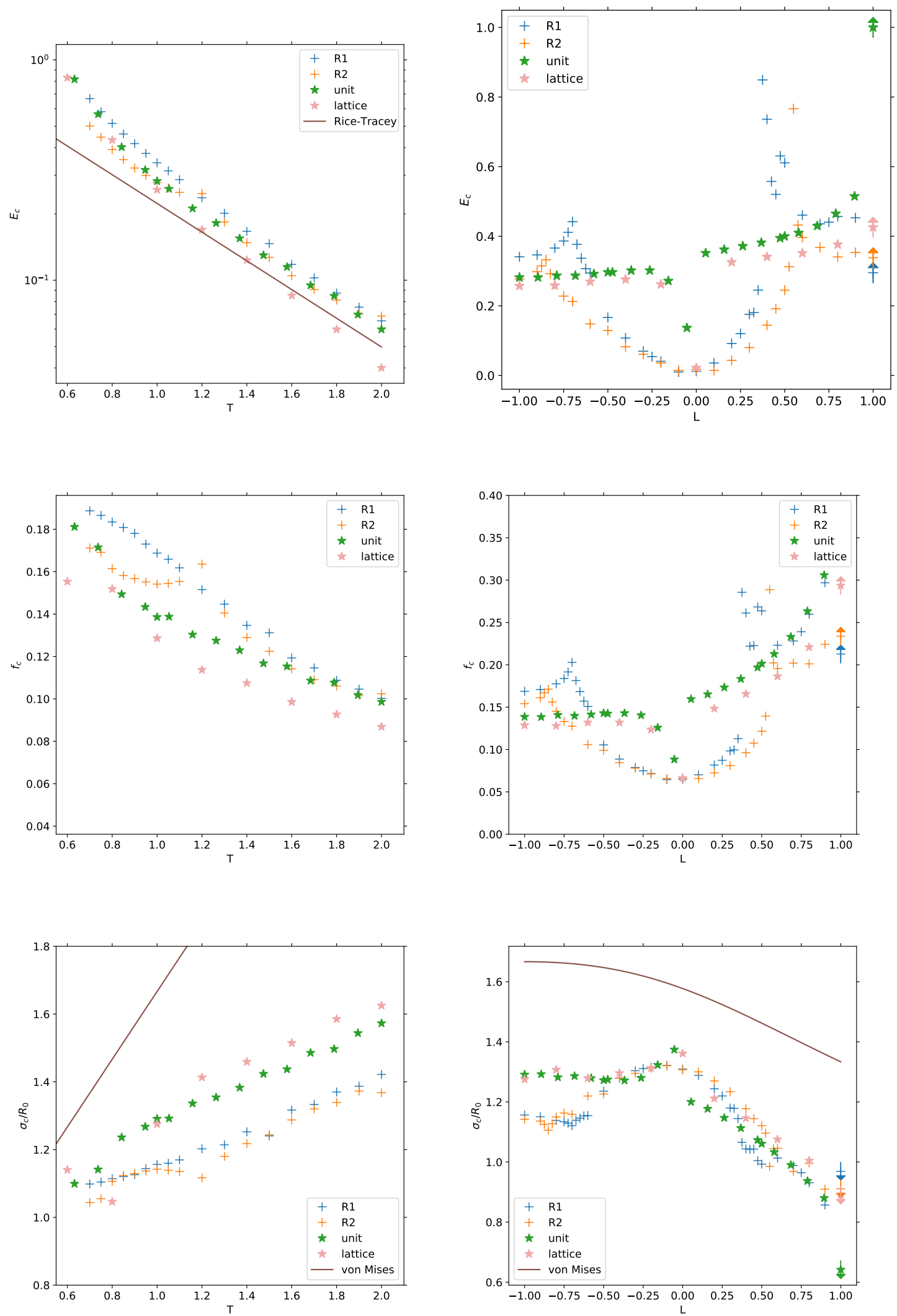

Fig. 3: Evolution of strain $E_{c}$ (top), porosity $f_{c}$ (center) and stress $\sigma_{c}$ (bottom) at coalescence for various microstructures, with respect to $T$ in generalized tension $L=-1$ (left column) or with respect to Lode parameter, at constant triaxiality $T=1$ (right column). The points with arrows at $L=1$ (right column) correspond to the last data point from simulations that diverged or for which the indicator was not reached: these points correspond to lower bounds (for $E_{c}$ and $f_{c}$ ) and upper bounds (for $\sigma_{c}$, as $\bar{\sigma}_{11}$ is decreasing with $E_{11}$ ) for the values at failure, if it does exist. 
rate varies as $\exp (3 T / 2)$. If we assume that coalescence happens at a given porosity (as for Tvergaard and Needleman (1984)), $E_{c}$ should vary as $\exp (-3 T / 2)$. The evolution of strain at coalescence for the random microstructures, the unit and the lattice cell can be well represented by this simple relation, as shown by the comparison with the straight line of slope $-3 / 2$.

The evolution of failure-related quantities with respect to triaxiality, at fixed $L=-1$ is thus very similar for the various studied microstructures, although some differences are visible. The situation is different if the triaxiality $T=1$ is fixed and the coalescence behavior is studied with respect to the Lode parameter (the whole range $L \in[-1,1]$ is explored). The results of the simulations are shown on the right side of figure 3 . Values for $L=1$ are indicated with superimposed arrows but should be taken with caution because, for these loading cases, the simulations diverged or the failure indicator was not reached; the data for the last computed point is indicated to serve as lower or upper bounds for the real value at coalescence, if it exists. The case $L=1$, which corresponds to an axymmetric loading where the two largest principal stress components are equal, is associated by Gologanu et al. (2001) to the necklace coalescence. Our criterion described in section 3 is not able to represent this type of coalescence, which is not associated to a loss of full rank of the deformation gradient rate. Examining the stress strain curve of the unit cell in the case $L=1$ (not shown here for brevity) shows a stabilization of stress which could be linked indeed to a coalescence event, undetected by the $\delta$ indicator.

If we do not consider anymore the values for $L=1$, the unit and lattice cells behave in a similar way (the difference between these two types of cells, which should represent the same void configuration, is due to the meshing): $E_{c}$ increases slowly with $L$. This type of evolution was reported by Zhu, Ben Bettaieb, et al. (2020) and Zhu, Engelhardt, et al. (2018) and by Guo and Wong's (2018) localization indicator (when $\bar{\sigma}$ does not have shear components). However, Barsoum and Faleskog (2011), Wong and Guo (2015), Dunand and Mohr (2014), Guo, Ling, et al. (2020), Zhu, Engelhardt, et al. (2018), and Guo and Wong (2018) (for the latter two, in more general loading cases), report that $E_{c}$ is a convex function of $L$, with a minimum near $L=0$. Yet, in our case, a sharp decrease in ductility is observed for $L$ close to zero. This behavior in generalized shear corresponds to the expected behavior of a perfectly plastic von Mises material which localizes immediately in shear.

However the random microstructures $R 1$ and $R 2$ do not exhibit the same evolution as the unit cell. Three zones can be observed on the $E_{c}-L$ plot for the $R 1$ microstructure (schematized in figure 4 ). The first zone corresponds to $L \in[-1,-0.7]$, in which $E_{c}$ increases up to a maximum value on a cusp. For $L \in[-0.7,0.4], E_{c}$ is convex in $L$ and minimal for $L=0$. The third zone corresponds to $L \in\left[0.4,1\right.$ [, where $E_{c}$ decreases from its maximum at $L=0.1$ and stabilizes. The zone boundaries correspond to local maxima (significantly higher than the rest of the data points) of $E_{c}$. They are associated to slope discontinuities, although $E_{c}$ remains continuous. 
In the following, these three zones will be referred to as: Low Lode parameter Extension Mode Zone (LLEMZ),

Shear Mode Zone (SMZ) and High Lode parameter Extension Mode Zone (HLEMZ); the rationale behind these names will be made clearer after section 4.2. A similar decomposition in three zones can be seen for the microstructure $R 2$ although for different zone boundaries $(L=-0.9$ and $L=0.55)$. To the knowledge of the authors, such an evolution of $E_{c}$ with respect to $L$ was not found in literature. Although Guo and Wong's (2018)'s coalescence criterion yields a non-smooth evolution of $E_{c}$, there is only one local maximum, for $L>0$ (with our definition of $L$ ). An explanation for the existence of these three zones will be proposed in section 5.1.

An asymmetry between positive and negative values of $L$ can also be observed: ductility $E_{c}$ is higher in generalized compression than in tension. This asymmetry is present in previously mentioned studies, but the sign of the difference varies among them. Our results are consistent with Zhu, Ben Bettaieb, et al. (2020) and Dunand and Mohr (2014) but Barsoum and Faleskog (2011), Wong and Guo (2015), and Guo and Wong (2018) have found cells more ductile in generalized tension than in compression (taking into account the different definitions of $L)$.

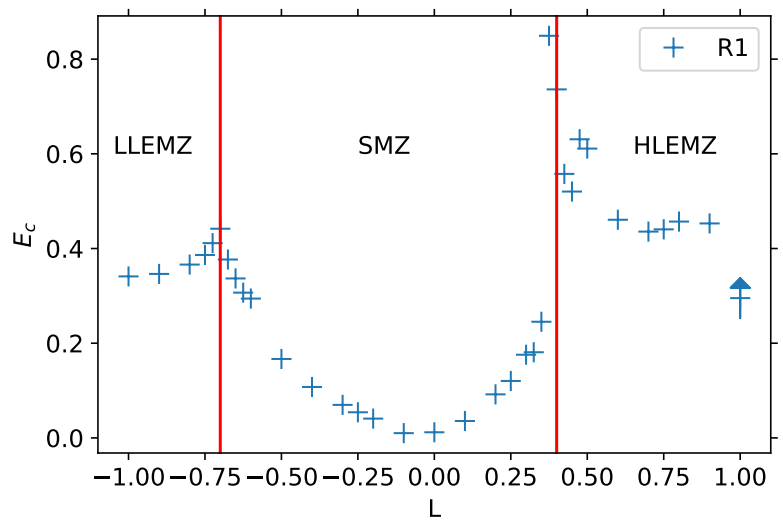

Fig. 4: Identification of three ductility zones, with respect to $L(R l$ microstructure, constant triaxiality $T=1)$.

Similar behaviors and differences between the unit and lattice cells on the one hand and the random microstructures on the other hand can be observed on the results for porosity at coalescence. For the stress at coalescence, the asymmetry between $L<0$ and $L>0$ is clear. There is no significant difference between the microstructures at $L>0$, for $L<0 ; \sigma_{c}$ is almost constant for the lattice and unit cells, whereas it increases slightly with $L$ for the random microstructures. No zone boundaries can be easily identified. The theoretical values for $\sigma_{c}$ obtained for a von Mises material failing when $\sigma_{v m}=R_{0}$ are also represented. The type of evolution 
agrees with the results for the unit cells, but due to the porosity and the complex coalescence behavior, stress

levels are significantly lower for the cells, and the slope of $\sigma_{c}$ with respect to $T$ for the simulations at constant $L=-1$ also differs.

In contrast to the unit and lattice cells, the random microstructures display several zones on their $E_{c}-L$ curve, which could be linked to different coalescence behaviors. The different zones for the microstructure $R I$ are also shown in the $T-L$ space in figure 5. Multiple simulations were carried out for $T \in[0.7,1.1]$. A simple interpolation using Gaussian Process Regression (as implemented in Scikit-learn (Pedregosa et al., 2011)) is proposed and allows for an easier visualization in the $E_{c}-T-L$ space, although cusps at zone boundaries are smoothed. The results of the simulations are also projected in the $E_{c}-L$ plane. The triaxiality has two effects on $E_{c}: E_{c}$ globally decreases with higher triaxiality levels, in agreement with the previous study at fixed $L=-1$, and the position of the zone boundaries is modified (at $T=1.1$, the central zone is wider than at $T=0.7$ ).
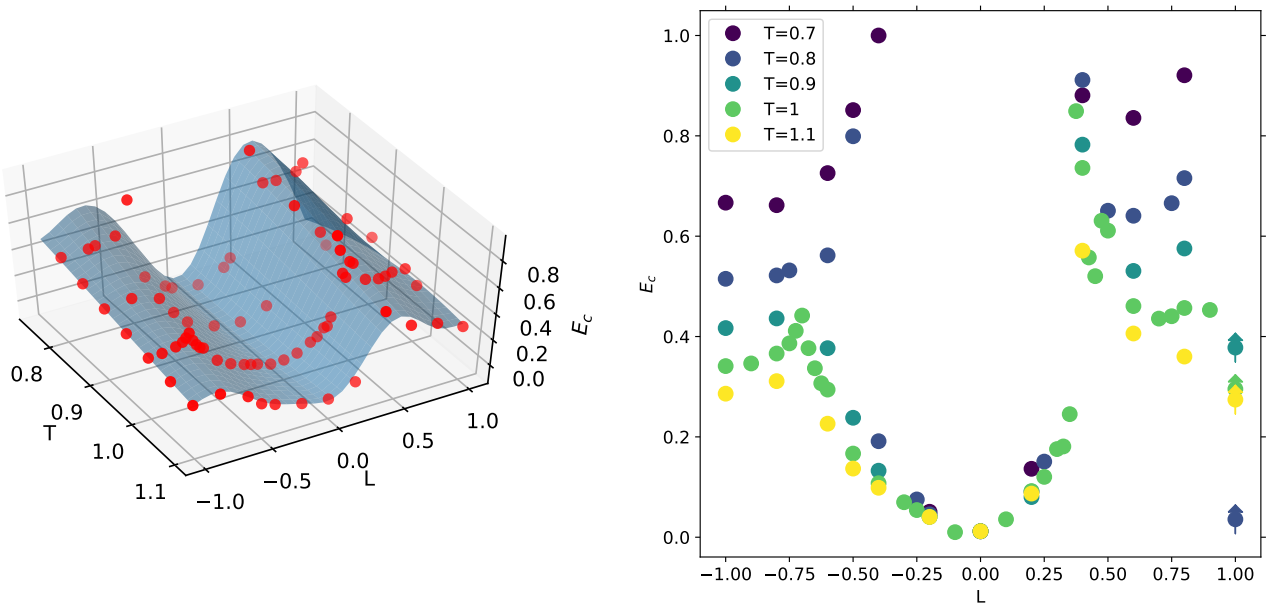

Fig. 5: Strain at coalescence in the $T-L$ space, for the $R 1$ microstructure. Left: Coalescence surface interpolated by Gaussian process regression for multiple loading cases (simulations shown as red points). Right: Projection of the simulation results on the $L-E_{c}$ plane. 4.2 Relation to localization modes

${ }_{413}$ Several ductility zones were identified on the strain at failure curves for the random microstructure cells, in 414 contrast to unit cells. However the $E_{c}$ curves only give macroscopic information and shed no light on the 
mechanisms inside the cell responsible for the drastic changes in strain at coalescence. We now investigate the relation between the presence of these zones and the aspect of strain fields inside the cell.

The figure 6 shows, for each microstructure, the cumulative plastic strain field $p$ shortly after coalescence (for $E_{11} \simeq 1.1 E_{c}$ ). Each image corresponds to a different $L$ value ( $T=1$ is fixed). The images are inserted on $E_{c}-L$ curves in order to better correlate macroscopic and field information.

All the $p$ fields display zones of higher strain or even strain localization (as localization is known to happen before coalescence (Guo and Wong, 2018)). These zones are organized along approximately planar bands. For both the unit and lattice cells, these bands are exactly planar and correspond to a crystallographic plane of the void lattice. In the lattice cells, the three rows of voids are equivalent, but this symmetry is broken after coalescence. For random microstructures, the bands are more complex: a base plane can be identified but bands are distorted by void distribution so as to include more voids.

For a given microstructure, the orientation of the bands is not constant with $L$. Two different orientations can be distinguished. In the first one the band is roughly parallel to a face of the cell (and perpendicular to the main loading axis). For the cases with $L \simeq 1$, the localization pattern is more complex and is composed of several bands. The second type of orientations is characterized by strain bands of overall direction approximately $45^{\circ}$ relative to the faces of the cell (although their precise shape is more complex). These two orientations are partly constrained by the periodic boundary conditions because strain localization bands should be compatible with the periodicity of the cell. Notice that bands oriented at $45^{\circ}$ are only found for Lode parameters close to zero (and only for $L=0$ in the regular unit and lattice cells) whereas parallel orientation is found for higher values of $|L|$. Observing more carefully the relation between the orientation of the bands and the macroscopic $E_{c}-L$ curves for the random microstructures shows that strain band orientation is systematically associated with ductility zones: the $45^{\circ}$ orientation is only found in the SMZ whereas parallel orientations are found in the LLEMZ and HLEMZ. Therefore the transition between ductility zones can be linked to a change in strain localization mode: between extension mode, with strain bands at parallel orientation, and shear mode characterized by the $45^{\circ}$ orientation.

To better characterize the transition between ductility zones, as explained by $p$ fields, the similarity between the $p$ field at coalescence for a given value of $L$ and three reference coalescence $p$ fields for $L \in\{-1,0,0.9\}$ is here quantified for the $R 1$ microstructure. Each loading case is considered a paragon of its ductility zone (respectively the LLEMZ, the SMZ, and the HLEMZ). If two $p$ fields are similar, they should represent a similar coalescence mechanism. A similarity indicator is defined as follows. The $p$ field after coalescence (at strain $\left.E_{11}=1.1 E_{c}\right)$, as produced by a FEM computation, is represented by the vector $[P]$ of $p$ values at all Gauss 


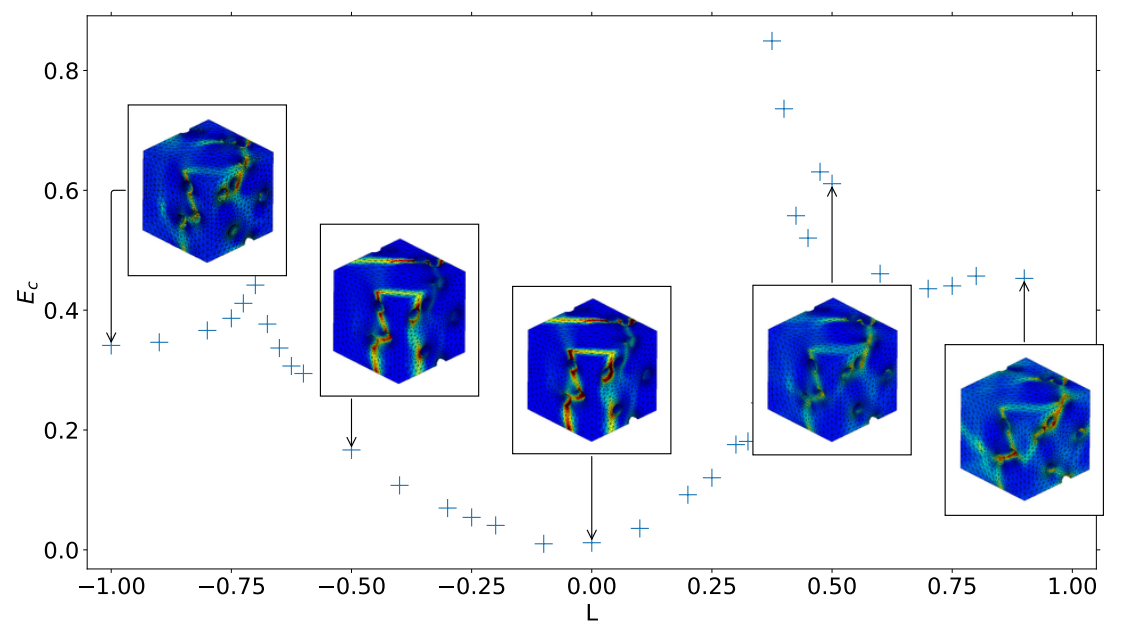

(a) Microstructure $R 1$

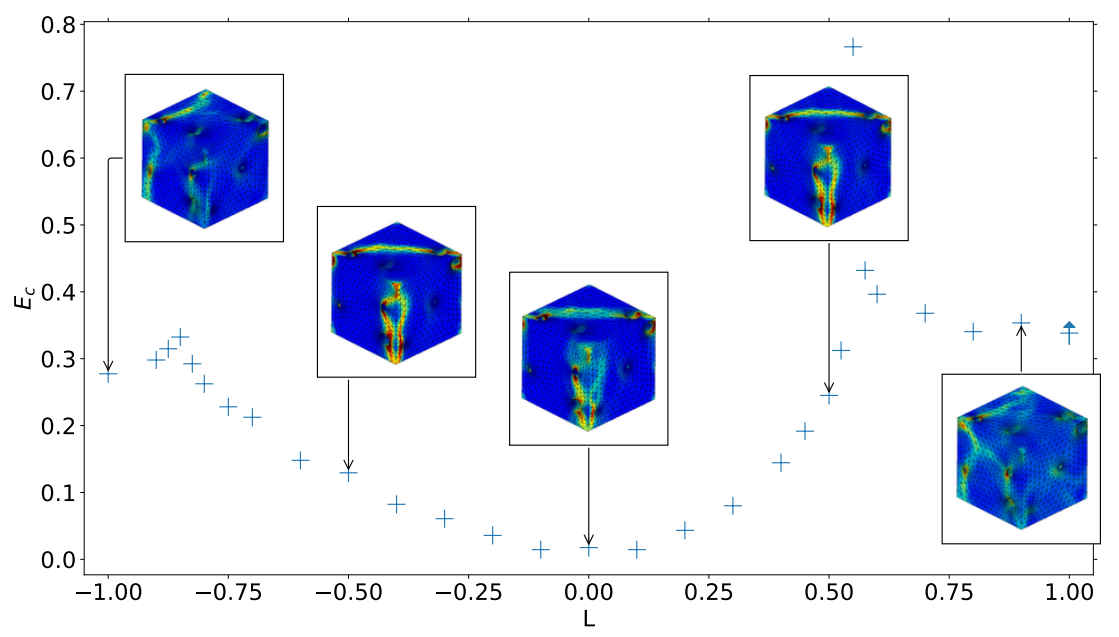

(b) Microstructure $R 2$

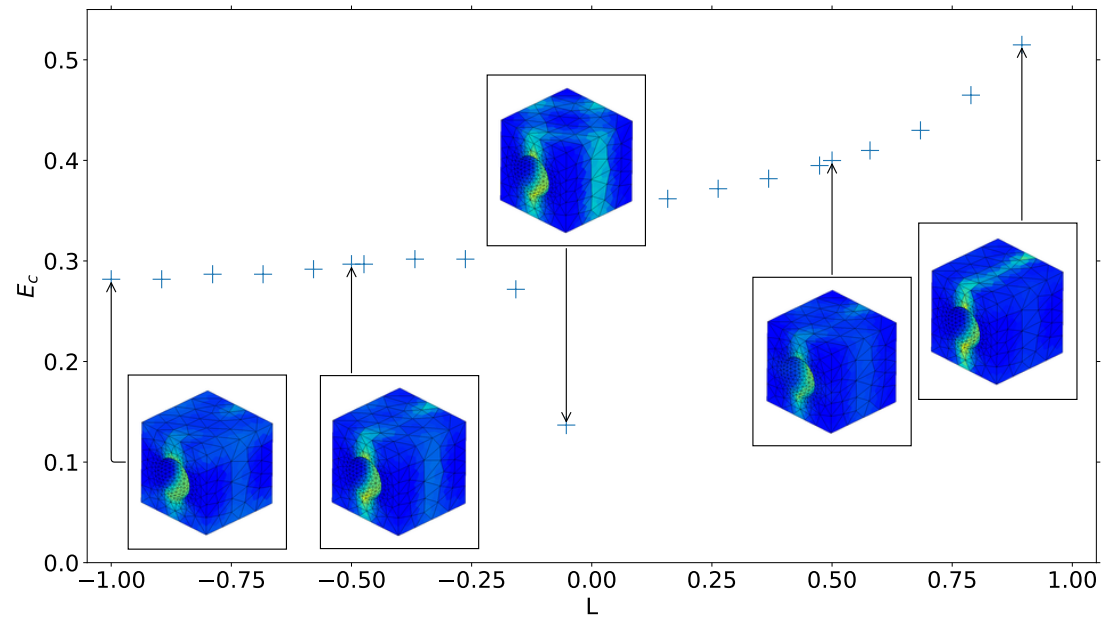

(c) unit cell 


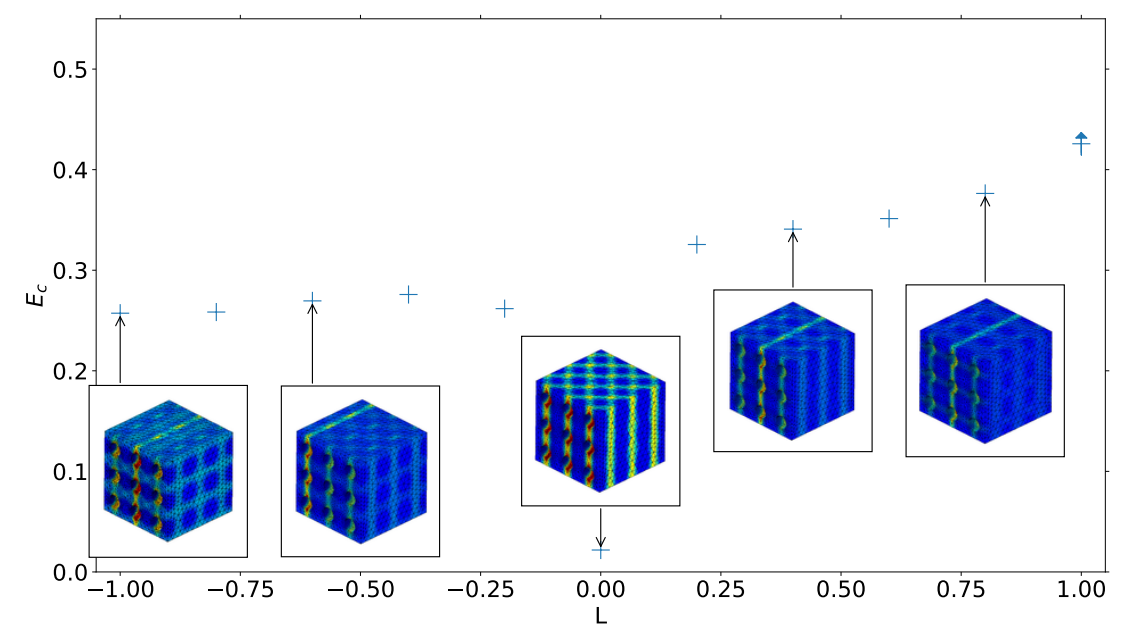

(d) Microstructure lattice

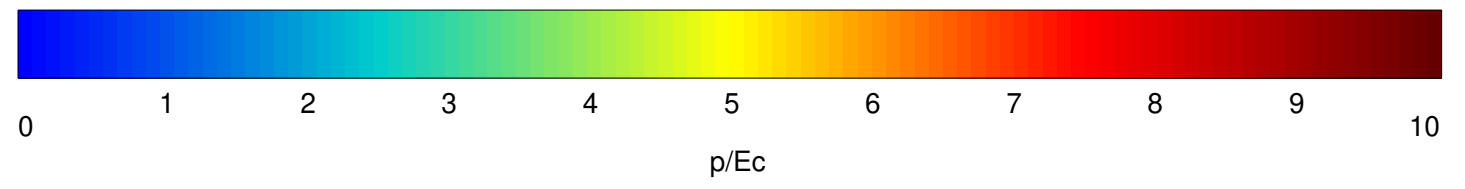

Fig. 6: Link between $E_{c}$ evolution with respect to $L$ and cumulative plastic strain field shortly after coalescence (at strain $E_{11}=1.1 E_{c}$, which depends on the simulation). Fixed triaxiality $T=1$

points (for the $R 1$ microscructure, the $[P]$ vectors are around $7 \times 10^{5}$ components long). The relative spatial position of Gauss points is irrelevant here. For two vectors $[P]$ and $\left[P^{\prime}\right]$ representing $p$ fields on the same mesh and with the same ordering of Gauss points, the similarity can then be defined as the angle (or rather its cosine) between $[P]$ and $\left[P^{\prime}\right]$ :

$$
\cos \left(\theta_{P P^{\prime}}\right)=\frac{[P] \cdot\left[P^{\prime}\right]}{\|[P]\| \cdot\left\|\left[P^{\prime}\right]\right\|}
$$

with $\|[P]\|$ the standard euclidean 2-norm of $[P]$. If $[P]$ and $\left[P^{\prime}\right]$ are proportional, $\cos \left(\theta_{P P^{\prime}}\right)=1$. This quantity is extracted from Z-set computations using tools developed by Lacourt et al. (2020).

The evolution of the similarity indicator $\cos (\theta)$ to the reference strain fields $L=-1, L=0, L=0.9$ is plotted in figure 7. The three reference strain fields are not orthogonal, so significant overlap between the indicators is possible. As strain fields at $L=-1$ and $L=0.9$ are similar $(\cos (\theta)=0.85)$, their similarity indicator shows comparable behavior. However the evolution of the indicator for $L=0$ is reversed. The three ductility zones defined earlier are apparent on the figure. For the LLEMZ $L \in[-1,0.7]$, the contributions of $L=-1$ and $L=0.9$ are high and almost constant whereas the contribution of $L=0$ is lower but increasing. On the contrary, in the 
458

SMZ $[-0.7,0.5]$, strain fields are predominantly linked with $L=0$ and little with $L=-1$ or $L=0.9$. In the last zone, HLEMZ, above $L=0.5$, the similarity to the $L=0$ strain field decreases, whereas $L=-1$ and $L=0.9$ contributions are higher. Notice however that the $L=-1$ similarity indicator is high at $L=0.5$ and decreases with $L$, unlike the $L=0.9$ indicator. For $L \simeq 0.5$, the situation is close to that of $L=-1$, whereas at very high $L$, another mechanism could come into play, especially the competition between two perpendicular strain bands observed earlier at very high $L$. Around the ductility zone boundaries, strain fields quickly change from one mode to the other. This competition between modes could explain the cusps in strain at failure observed at zone boundaries.

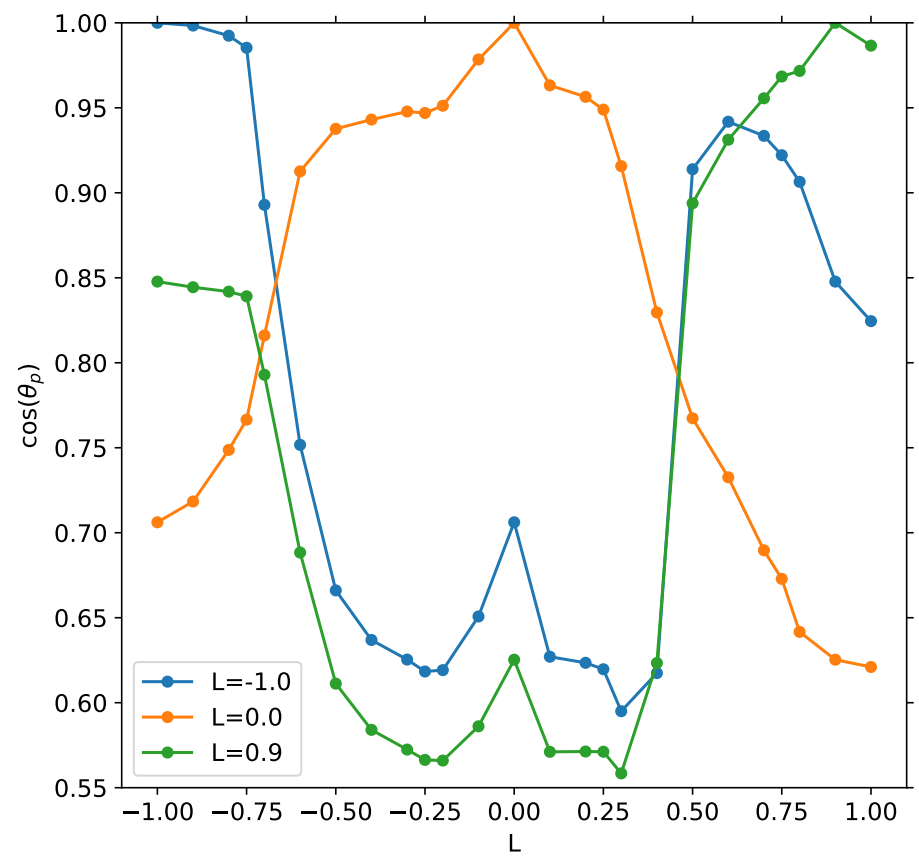

Fig. 7: For the $R 1$ microstructure, similarity between the coalescence $p$ field at varying $L(T=1$ is fixed) and three reference $p$ fields obtained at $L=-1, L=0, L=0.9$. 
4.3 Dispersion due to microstructure sampling

In the previous two sections, two random microstructures were considered and the evolution of coalescencerelated quantities with respect to loading conditions were studied, showing significant differences when compared to the unit cell. Rather than choosing fixed microstructures and varying $T$ and $L$, another approach is to treat $E_{c}$, $f_{c}$ and $\sigma_{c}$ as random variables (depending on the microstructure), and study their statistics.

$N=20$ microstructures with 27 voids and initial porosity $f_{0}=6 \%$ ( $f_{0}$ is not a random variable) were randomly and independently generated. Each of them was subjected to the same loading conditions $(T, L) \in$ $\{(1,-1),(1,-0.5),(1,0.5),(1.5,-0.5)\}$. The results for $E_{c}, f_{c}$ and $\sigma_{c}$ are shown in figure 8 as box plots, and are compared to the values for the unit cell. A strong relative dispersion is present for all loading cases: the ratio of the standard deviation to the average is $34 \%, 59 \%, 55 \%$ and $62 \%$ respectively. This indicates a strong influence of the microstructure on the coalescence behavior. The results from unit cells do not represent well the behavior of the multiple void cells, and lead for instance to an overestimation of the stress at coalescence. Dispersion also depends on the loading conditions: for $T=1$, the case $L=-1$ shows lower interquartile range than the cases $L= \pm 0.5$. This can be linked to the proximity of zone boundaries for the latter cases, as $E_{c}$ was shown to vary sharply near those boundaries. Moreover, and especially for $L=0.5$, some microstructures coalesce in tensile mode whereas others coalesce in shear mode (compare for instance the strain fields of $R I$ and $R 2$ in figure 6); the possibility of different coalescence modes may increase dispersion. At higher triaxiality $T=1.5, L=-0.5$, the dispersion is reduced for $E_{c}$ and $f_{c}$ when compared to $T=1, L=-0.5$ but the relative dispersion is not. This is due to the overall effect of coalescence appearing earlier at high triaxiality. Besides, the interquartile range for $\sigma_{c}$ is comparable for both triaxiality levels.

The previous results dealt with a small number of loading conditions. In order to determine an effective model of coalescence in random multiple-void cells for all loading conditions, the $T-L$ space should be explored more extensively, while still keeping a large enough set of microstructure realizations. As in section 4.1, multiple simulations were carried out for $T \in[0.7,1.1]$ and $L \in[-1,1]$ on five random microstructures among which $R I$ and $R 2$ (keeping 20 realizations would have been computationally too expensive). The same loading conditions were tested for each microstructure. The results for $E_{c}$ are shown in figure 9. The minimal, maximal and average values are first plotted for $T=1$. In agreement with the preceding results, significant relative dispersion is present, and its extent depends on $L$ : dispersion is particularly strong near $L=0.5$, whereas it is negligible for $L=0$ (all the microstructures agree on almost immediate localization for generalized shear). Despite the dispersion, the overall aspect of the $E_{c}$ curve, as described in the previous section, and its decomposition in 

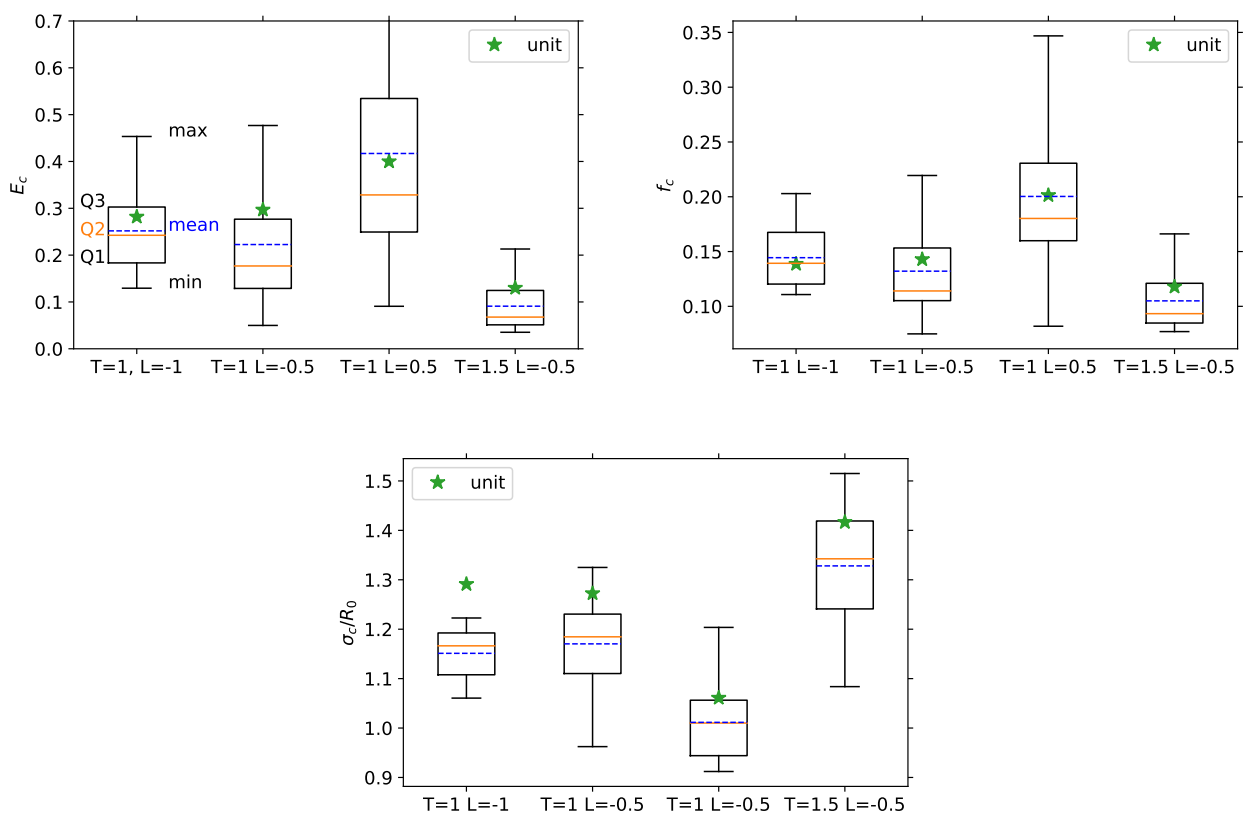

Fig. 8: Dispersion of strain, porosity and stress at coalescence for different loading conditions, when considering multiple $(\mathrm{N}=20)$ random populations of 27 defects $(\star$ : comparison with the results for unit cell)

ductility zones, are still observable. An interpolation by Gaussian Process Regression of the results in the $T-L$

space is also proposed, based on the average value of the five microstructures at each loading conditions. The aspect is similar to that of figure 5 .
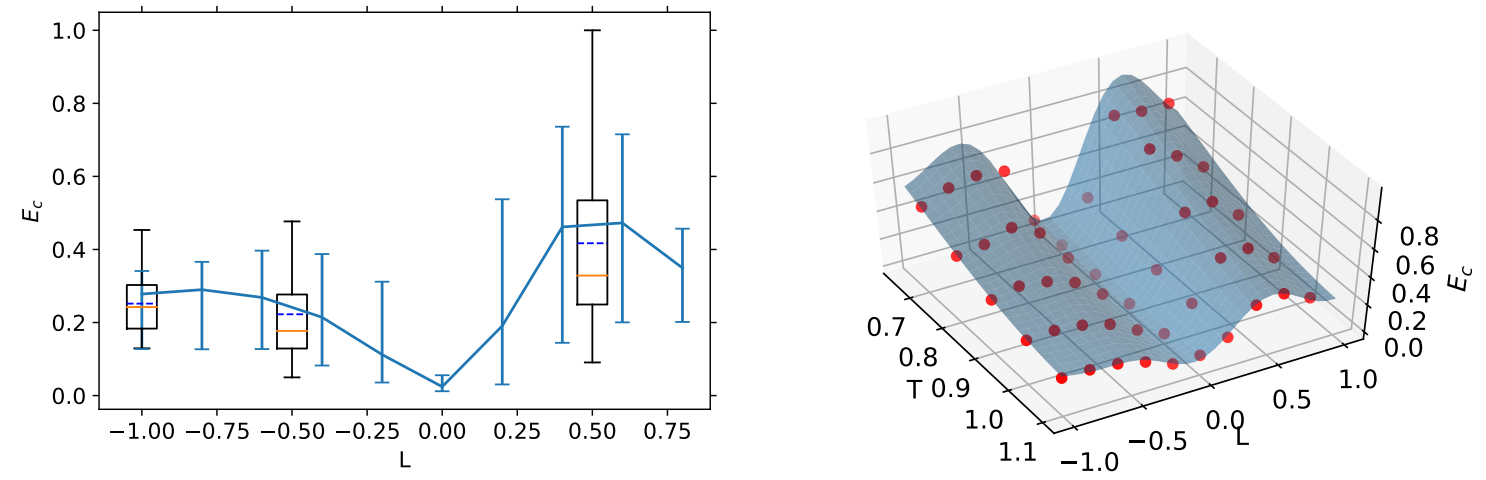

Fig. 9: Strain at coalescence results for five different microstructures. Left: For $T=1$, evolution of the average, minimum and maximum value of $E_{c}$ (over the size-5 sample) with respect to $L$ (earlier results from 20 realizations are also plotted). Right: averaged behavior with respect to $T$ - $L$ interpolated using Gaussian Process Regression. 


\section{Discussion}

In this section, we discuss the significance of the results presented up to now, and assess how representative the results are and how far they can be generalized. First we compare the failure indicator proposed in section 3 to Wong and Guo's (2015) coalescence criterion, in order to interpret the difference between unit and random cells. The dispersion due to the random microstructures is then statistically studied with increasingly large void populations. Finally the influence of a work-hardening material is also addressed.

\subsection{Interpretation of the proposed failure indicator}

In order to better understand the failure mechanism identified by the $\delta$ indicator and the observed difference between the unit cells and the random microstructures, the $\delta$ indicator is compared to another failure criterion reported in the literature. We focus on Wong and Guo's (2015) energy-based coalescence indicator, although Zhu, Ben Bettaieb, et al.'s (2020) and Dæhli et al.'s (2020) approach with Rice's (1976) criterion could also be useful. According to the former indicator, coalescence is associated to concentration of the plastic deformation in the ligament whereas elastic unloading takes place elsewhere. Therefore coalescence can be detected by monitoring the evolution of the plastic $\dot{W}_{p}$ and elastic $\dot{W}_{e}$ work rates and the onset corresponds to the minimum of the ratio $\dot{W}_{e} / \dot{W}_{p}$.

For our cells, the corresponding work rates can be computed by the following equations:

$$
\begin{aligned}
\dot{W}_{p} & =\int_{V} \sigma_{v m} \dot{p} \mathrm{~d} V \\
\dot{W}_{t o t} & =V_{0} \underset{\sim}{\bar{S}}: \dot{\overrightarrow{\bar{F}}} \\
\dot{W}_{e} & =\dot{W}_{t o t}-\dot{W}_{p}
\end{aligned}
$$

The plastic power can be computed either on the cell (with voids) or more easily on the matrix, since stress is zero in the voids. The total power, sum of the plastic and elastic parts, can be computed by only using macroscopic quantities, according to the results of homogenization theory (Besson, Cailletaud, et al., 2009).

The figure 10 compares the failure onsets, as determined by the $\delta$ and the energy-based criteria, with respect to $L$, for the unit cell and the microstructure $R 1$. For the unit cells, the energy criterion identifies a coalescence onset for all the simulations, and the trend is typical of $E_{c}$ vs. $L$ curves in the literature (for instance Zhu, Ben Bettaieb, et al. (2020)). Moreover the two criteria yield similar values of $E_{c}$, except for $L=0$ where the $\delta$-criterion predicts early failure as previously. The situation is more complex for the random microstructure. For 


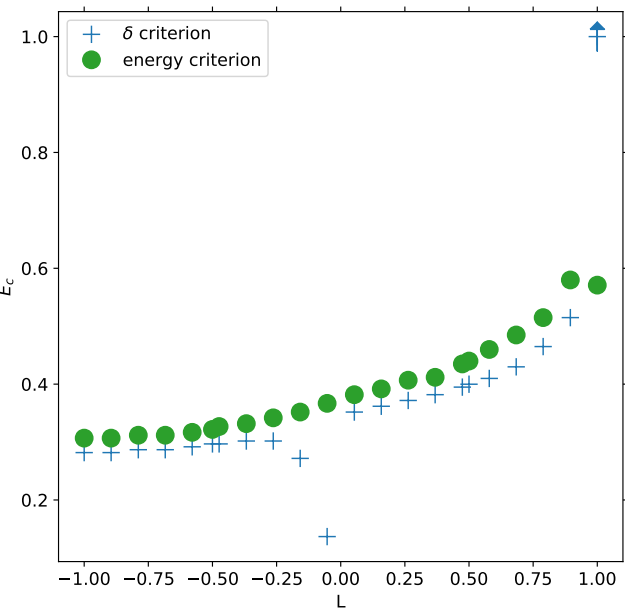

(a) Unit cell

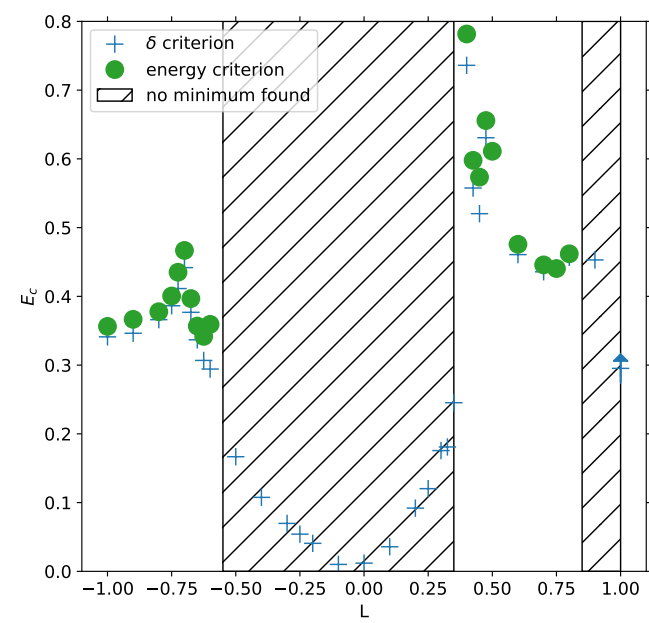

(b) Random microstructure $R 1$

Fig. 10: Comparison of the coalescence onset, as determined by the $\delta$-indicator and the energy-based criterion. All computations at triaxiality $T=1$. The hatched zones correspond to simulations for which no minimum of $\dot{W}_{e} / \dot{W}_{p}$ was observed, and therefore no coalescence was identified by the energy-based criterion.

The existence of the three ductility zones and the lower ductility in the SMZ could actually be due to boundary conditions. As noted previously, two evolutions of $E_{c}$ with respect to $L$ are reported in the literature: in Barsoum and Faleskog (2011), Dunand and Mohr (2014), and Wong and Guo (2015), strain at coalescence is 


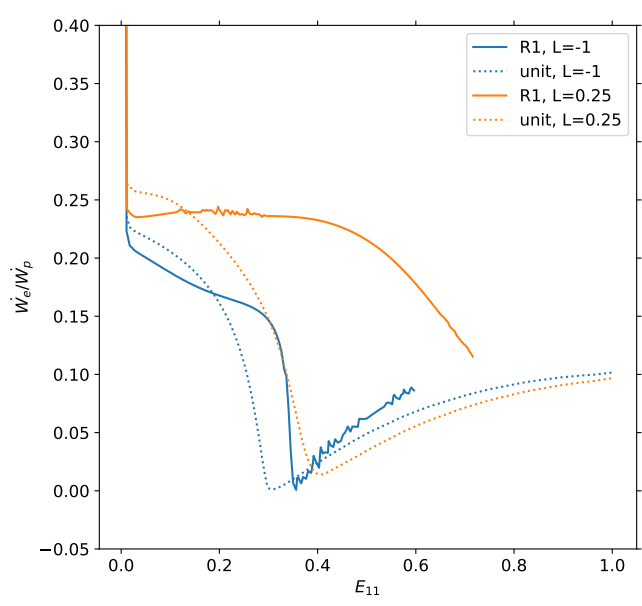

(a)

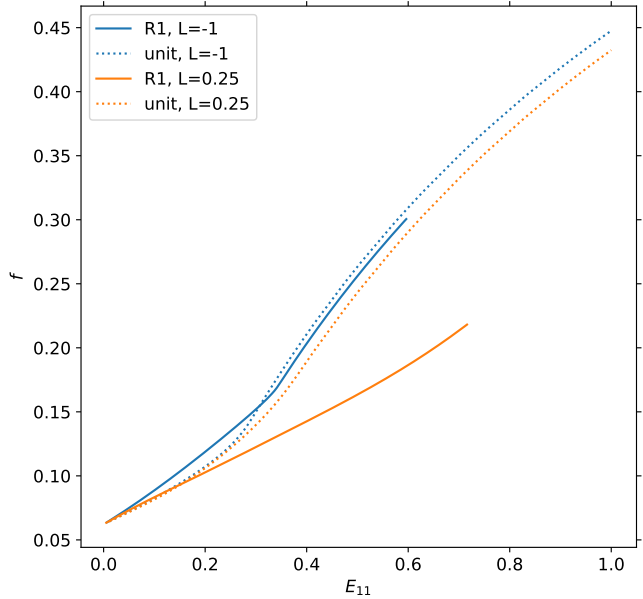

(b)

Fig. 11: Comparison of the evolution of power ratio $\dot{W}_{e} / \dot{W}_{p}$ (left) and of porosity $f$ (right), for the unit and $R 1$ cells in two loading cases: $T=1, L=-1$ and $T=1, L=0.25$

minimal for $L=0$ whereas it increases almost linearly for Zhu, Engelhardt, et al. (2018) and Zhu, Ben Bettaieb, et al. (2020). The difference between these two groups of studies is that the former consider a shear stress component in equation (13). Several loading conditions therefore correspond to the same triaxiality and Lode parameter, and the reported strain at coalescence is the minimum value over all tests at a given $(T, L)$ couple. Coalescence therefore happens earlier than in the absence of shear stress, and this might lead to different responses, as pointed by Zhu, Ben Bettaieb, et al. (2020). Another point of view is that the cubic unit cells have an anisotropic localization behavior. Although the cubic cell paves space when periodic boundary conditions are enforced, the axes parallel to the sides of the cube remain privileged, and the response of the homogenized material displays anisotropy. As localization bands should be compatible with the periodic boundary conditions, they are always parallel or around $45^{\circ}$ to one face of the cube (Coenen et al., 2012). Adding a shear stress component amounts to changing the principal loading directions relatively to the cube, and coalescence occurs when the most favorable band activates.

In the present study, shear stress was not considered but for the random microstructures, it was shown that coalescence can happen either by a localization band perpendicular (corresponding to the LLEMZ and HLEMZ) or oriented at $45^{\circ}$ to the main loading axis (for the SMZ). Therefore the random microstructures appear softer than the unit cells in that they allow several localization band orientations. The resulting response of the cell is then due to a competition between a limited number of coalescence modes (instead of the theoretical infinity of orientations considered by Barsoum and Faleskog (2011) for instance). The LLEMZ and the HLEMZ 
correspond then to the evolution shown by Zhu, Engelhardt, et al.'s (2018) study, whereas the response of random microstructures in the SMZ near $L=0$ is closer to that of unit cells in Barsoum and Faleskog (2011)'s study.

\subsection{Influence of the number of voids}

The microstructures considered in the above sections were composed of 27 voids. A small number of voids allows to investigate the effect of a cluster of pores whereas a sufficiently large number can provide results for an effective homogenized material. As pointed by Morin, Leblond, Benzerga, and Kondo (2016), the homogenization theory does not stricto sensu apply to coalescence, which takes place in a small area in the immediate vicinity of voids.

For computational homogenization with a volume element (VE) approach, random microstructures should contain enough voids to reduce the uncertainty due to sampling and limit the influence of boundary conditions (as there is no intrinsic length scale, the size of the VE is only determined by the number of voids it contains). However the computation power required to simulate large cells with many voids, which lead to FEM problems with millions of degrees of freedom, is prohibitive if carried on dozens of loading conditions and microstructures. This problem is in part mitigated by the use of periodic boundary conditions: Kanit et al. (2003) showed that homogenized properties converge faster with VE size in this case than with kinematic or static uniform boundary conditions. Their study dealt however with elasticity and the extrapolation to coalescence properties is not possible yet. Hure (2021), who carried out simulations of cells with random voids up to coalescence, compared cells with different number of voids (up to 64) and reported that the maximum stress reached during the simulation stabilizes with the number of voids (indicating the existence of a representative volume element), but the stress at coalescence still shows dispersion between realizations. However only five simulations were performed for each number of voids, which is limiting for a statistical analysis of dispersion.

In a complementary approach, we compare the strain at coalescence results for cells with different numbers of voids: $27,64,125$. All cells are generated with the process described in section 2.1 and their porosity is always $6 \%$; the meshing parameters are however adapted so that the ratio between void radius and maximum element size remains constant for all cells. There are typically $2 \times 10^{5}, 6 \times 10^{5}$ and $1 \times 10^{6}$ nodes for meshes of cells embedding 27, 64 and 125 voids respectively. As the computational cost of the simulations increases with the number of voids, we only considered two loading conditions $T=1, L=-1$ and $T=1, L=-0.5$ and a smaller number of 125 -void cells than the twenty 27 -void cells already used in section 4.3. Examples of $p$ fields 
after coalescence for a microstructure with 125 voids (fig. 12) display very complex localization paths between voids, but still show a principal direction parallel to or at $45^{\circ}$ from the faces.

Dispersion results are shown in figure 13. For the $T=1, L=-1$ case, dispersion is comparable for the three types of cells: a Brown-Forsythe test (Brown and Forsythe, 1974) was carried out to verify the equality of variances for the 27, 64 and 125-void groups of cells (this test and the following one use the Scipy implementation (Virtanen et al., 2020)). The statistical p-value is 0.19 so the hypothesis of equal variances cannot be rejected. The mean failure strain is significantly lower for 64 and 125-void cells than for 27-void cells, as proven by a one-way ANalysis Of VAriance (Heiman, 2001) between the three groups (p-value of 0.002). However for the $T=1, L=-0.5$ loading case, the dispersion is significantly lower for the 64-void cell (Brown-Forsythe test between the three groups: p-value of 0.026). The average failure strain seems to decrease with the number of voids (an ANOVA test could not be performed due to the unequal variances)

Therefore failure seems to begin earlier for cells with more voids. This could be explained by the higher probability of a favorable path for a localization bands when the number of voids grows. Variance remains high for all groups of cells, but it is possible that the number of voids reduces dispersion. The simulations evidence that the size of the volume element can exert an influence on the failure results. The above simulations therefore extend Hure's (2021) study with the results from larger and more numerous cells (allowing a statistical analysis) and are in agreement with his findings. More simulations at an even higher number of voids could be carried out to reinforce the statistical significance of the previous conclusions.

\subsection{Influence of material behavior}

The results previously described hold for a perfectly plastic material. However hardening can mitigate the effects of softening due to void growth, and delay coalescence. We here consider two other types of material behavior characterised by their flow stress functions $R(p)$ replacing the constant $R_{0}$ used for perfect plasticity in equation (4):

$$
\begin{array}{lr}
R(p)=R_{0}^{\prime}+K p^{n} & \text { (power law hardening) } \\
R(p)=R_{\infty}-\left(R_{\infty}-R_{0}^{\prime}\right) \exp (-b p) & \text { (saturating exponential) }
\end{array}
$$

with $R_{0}^{\prime}=350 \mathrm{MPa}, R_{\infty}=500 \mathrm{MPa}, K=343.5 \mathrm{MPa}, n=0.58, b=10$ or $b=200$. The different yield functions are shown in figure $14 \mathrm{a}$. 


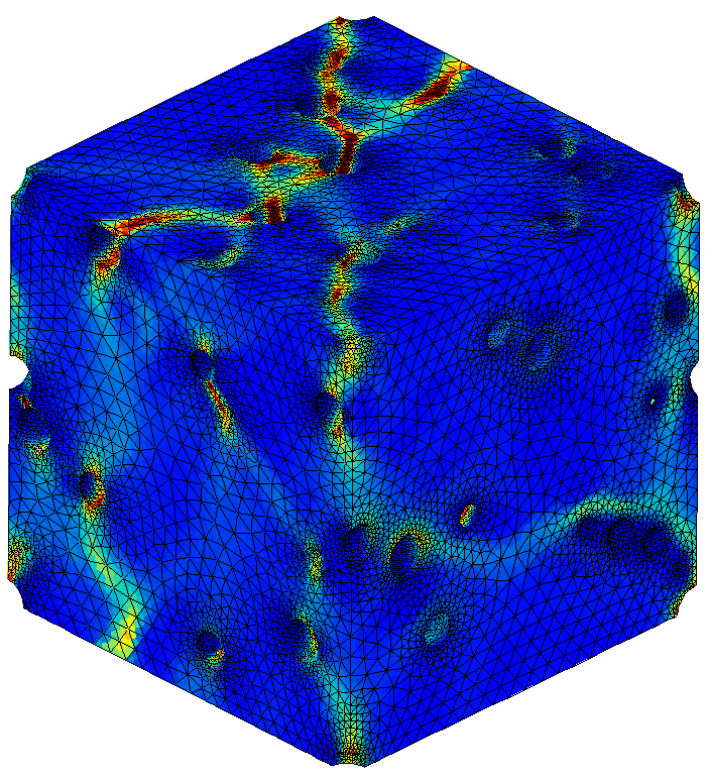

(a) $T=1, L=-1$

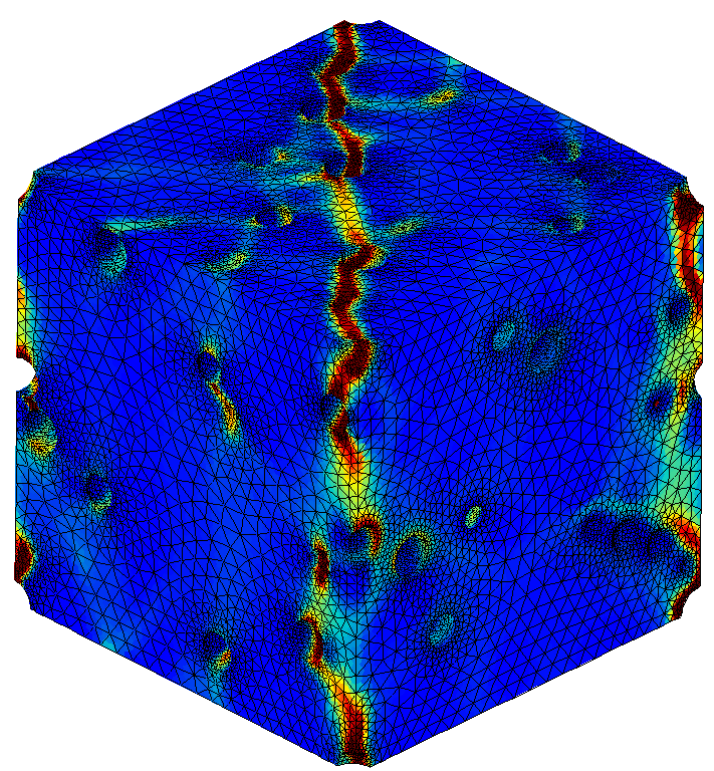

(b) $T=1, L=-0.5$

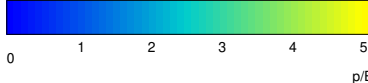

Fig. 12: Cumulative plastic strain fields after coalescence for a microstructure with 125 voids

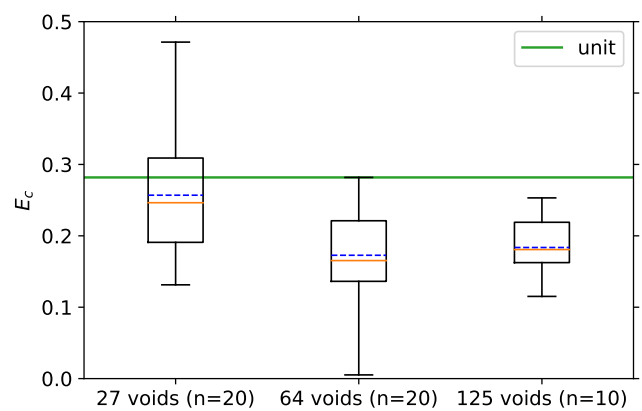

(a) $T=1, L=-1$

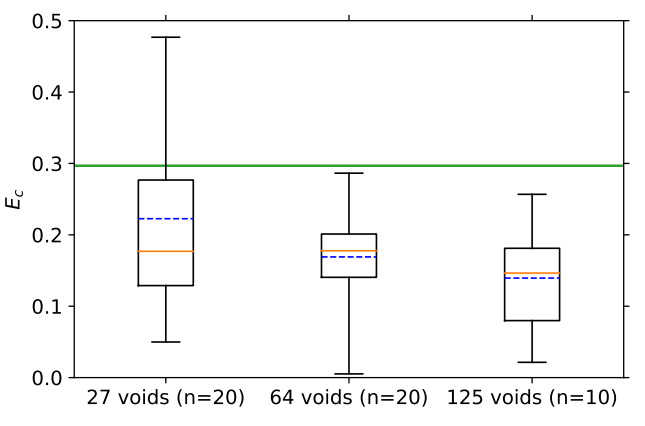

(b) $T=1, L=-0.5$

Fig. 13: Dispersion in the strain at coalescence for cells containing 27, 64, 125 voids, in two loading cases

For the $R 1$ microstructure, at fixed $T=1$ and varying $L$, a comparison of the strain at coalescence $E_{c}$ between the three hardening behaviors is shown in figure 14b. On the one hand, for the power law hardening and the slow saturating exponential hardening $b=10$, no central SMZ is observed (except a sudden drop near $L=0$ ), and the evolution is quite similar to that observed for unit cells in section 4.1. On the other hand, if hardening saturates more rapidly, as for $b=200$, the same response as in the perfectly plastic matrix case is 


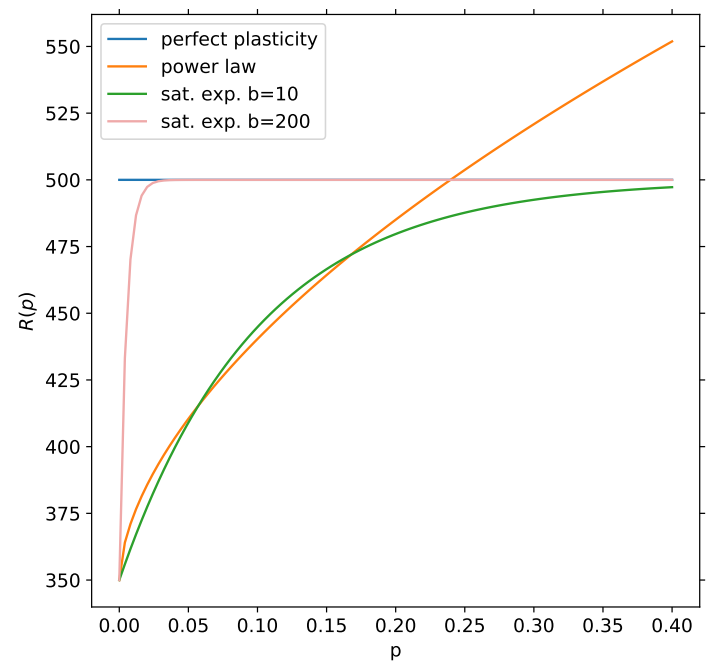

(a)

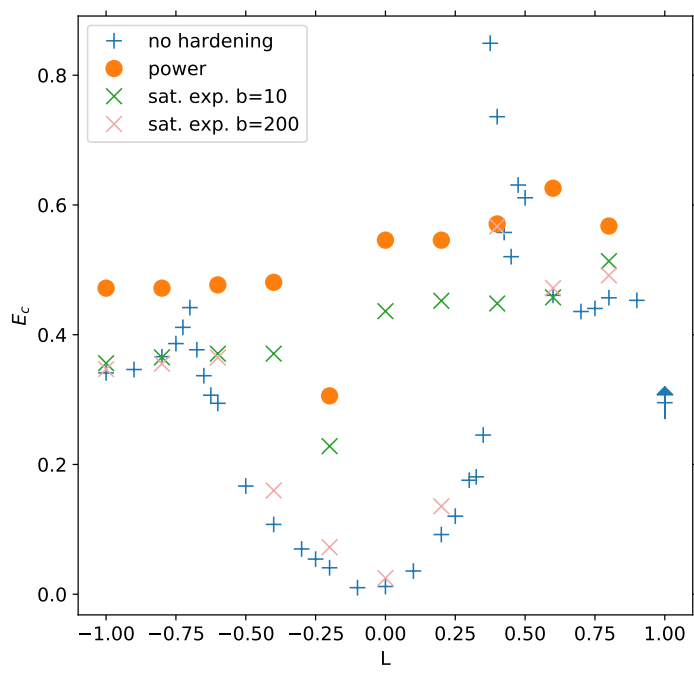

(b)

Fig. 14: Comparison of different hardening behaviors. (a): yield function for each hardening type. (b): Comparison of the $E_{c}-L$ curves for each hardening type on the microstructure $R 1$. All computations at fixed triaxiality $T=1$.

\section{Conclusion}

In the present study, random microstructures made of identical spherical voids within an elastoplastic matrix were generated, and simulated at constant stress triaxiality and Lode parameter with periodic boundary conditions.

The FEM simulations were carried out in a large strain framework up to coalescence. The major findings are the following:

1. Failure was identified using an indicator based on the loss of full rank of the average deformation gradient rate, while taking into account the response in case of homogeneous deformation. The results of this indicator are consistent with other indicators reported by the literature but better captures shear dominated localization modes.

2. Random microstructures show two failure modes, that differ by the orientation of the localization band: perpendicular to the main loading axis for an extension mode, or oriented around $45^{\circ}$ for a shear mode. 
Unlike unit and lattice cells, the shear mode is not limited to the immediate neighborhood of $L=0$. The competition between these two modes leads to a non-smooth evolution of the strain at coalescence with respect to the Lode parameter, showing three zones on the $E_{c}-L$ curve, with reduced ductility near $L=0$. The difference between unit cells and random microstructures is reduced when the matrix is no more perfectly plastic, due to a stabilizing effect of hardening. However, the response with respect to $T$ is similar for unit cells and random microstructures.

3. When applying the same loading state to microstructures with similar characteristics, a significant dispersion is found in the results (up to $60 \%$ of relative dispersion for strain at coalescence). This strong dispersion is also found in simulations with a higher number of voids.

If a model expressing coalescence quantities with respect to loading conditions is desired, using unit cells therefore appears to misrepresent the effective behavior of a material with a complex void distribution, with differences in the general evolution and oversight of the statistical aspects. Care should therefore be taken when applying results on unit cells to more complex applications.

The present work could be extended in several ways. Firstly larger population sizes will be considered based on parallel computing, in order to improve the statistical representativeness of the presented results. Secondly a broader description of the mechanisms of coalescence in random microstructures will be reached by adding a macroscopic shear stress component to the loading state, so as to explore a greater variety of loading paths. Moreover the link between the proposed coalescence indicator and strain localization criteria such as macroscopic or local loss of ellipticity will also be investigated. Finally this work can be the basis to develop and calibrate an effective damage and plasticity model for materials containing randomly distributed pores. Hure (2021) proposed an example of such a homogenized model, but a new model could integrate the effects of the Lode parameter and the dispersion. However simulating enough loading cases and with sufficient statistical representativeness to completely explore the space of parameters is computationally expensive, especially as the effect of initial porosity should be taken into account. Therefore a strategy to construct a surrogate model with as reduced a number of required simulations as possible should be developed.

Acknowledgements The authors would like to thank L. Lacourt for valuable discussion and his help in the analysis of localization modes.

\section{Conflict of interest}

The authors declare that they have no conflict of interest. 
where $b_{2}$ and $b_{3}$ are functions to be determined. As $\underset{\sim}{\bar{D}}=\operatorname{sym}\left(\underset{\sim}{\dot{\bar{F}}} \bar{\sim}^{-1}\right)$, and $\underset{\sim}{\bar{F}}$ is diagonal, $\bar{\sim}$ can be written as:

$$
\underset{\sim}{D}=\underset{\sim}{\dot{F}} F^{-1}=\operatorname{diag}\left(\frac{\dot{\varepsilon}}{1+\dot{\varepsilon} t}, \frac{\dot{b}_{2}}{b_{2}}, \frac{\dot{b}_{3}}{b_{3}}\right)
$$

For a perfectly plastic Green material, the behavior law in (4) reads:

$$
\underset{\sim}{D}=\frac{\dot{p}}{\sigma_{e q}}\left(\frac{3}{2}{\underset{\sim}{\sigma e v}}^{d e}+(\operatorname{tr} \underset{\sim}{\sigma}) \underset{\sim}{1}\right)=\frac{\dot{p}}{\sigma_{e q}}\left(\frac{3}{2} \underset{\sim}{\sigma}+\left(C-\frac{1}{2}\right)(\operatorname{tr} \underset{\sim}{\sigma}) \underset{\sim}{1}\right)
$$

$\underset{\sim}{D}$ is diagonal so there are three constants $\alpha_{1}, \alpha_{2}$ and $\alpha_{3}$ such that:

$$
\begin{aligned}
\underset{\sim}{D} & =\operatorname{diag}\left(\alpha_{1}, \alpha_{2}, \alpha_{3}\right) \\
\alpha_{1}+\alpha_{2}+\alpha_{3} & =\frac{3 C \operatorname{tr} \underset{\sim}{\sigma}}{\sigma_{e q}} .
\end{aligned}
$$


Combining (25) and (28) yields the system:

$$
\begin{aligned}
\frac{\dot{\varepsilon}}{1+\dot{\varepsilon} t} & =\dot{p} \alpha_{1} \\
\dot{b}_{2} & =\dot{p} \alpha_{2} b_{2} \\
\dot{b}_{3} & =\dot{p} \alpha_{3} b_{3}
\end{aligned}
$$

667

668

The plastic multiplier is then $\dot{p}=\frac{\dot{\varepsilon} / \alpha_{1}}{1+\dot{\varepsilon} t}$ and the differential equations can be solved with the initial conditions $b_{2}(0)=1, b_{3}(0)=1$ :

$$
b_{2}=(1+\dot{\varepsilon} t)^{\alpha_{2} / \alpha_{1}} \quad b_{3}=(1+\dot{\varepsilon} t)^{\alpha_{3} / \alpha_{1}}
$$

Finally,

$$
\operatorname{det}(\dot{\sim})=\dot{\varepsilon} \dot{b}_{2} \dot{b}_{3}=\dot{\varepsilon}^{3} \frac{\alpha_{2} \alpha_{3}}{\alpha_{1}^{2}}(1+\dot{\varepsilon} t)^{\frac{\alpha_{2}+\alpha_{3}}{\alpha_{1}}-2}=\dot{\varepsilon}^{3} \frac{\alpha_{2} \alpha_{3}}{\alpha_{1}^{2}}(1+\dot{\varepsilon} t)^{-3-3 C \frac{\operatorname{tr} \sigma}{\sigma e q} \alpha_{1}}
$$

The function comparing the behavior of $\operatorname{det}(\underset{\sim}{\dot{F}})$ should and the homogeneous plastic deformation case is then:

$$
\delta_{C}(t)=\dot{\varepsilon}^{-3}(1+\dot{\varepsilon} t)^{3-3 C \frac{\operatorname{tr} \sigma}{\sigma_{e q} \alpha_{1}}} \operatorname{det}(\underset{\sim}{\dot{\bar{F}}})
$$

The $\delta$ criterion used throughout the article is recovered by setting $C=0$, which corresponds to the simplified case of a von Mises material. In this case, the criterion depends no more on the applied $\sigma$.

The evolution of $\delta$ for a simulation with $T=1$ and $L=-1$ (coalescence in uniaxial strain state) is shown in figure 15, for two values of $C: 0$ and $1 / 2$. For both values of $C$, the vanishing of $\delta_{C}$ is simultaneous with the stabilization of transverse displacement. However the sharp drop of $\delta_{C}$ allows a more precise numerical determination of the onset of coalescence than the more progressive stabilization of the transverse strain. For $C=1 / 2, \delta_{1 / 2}$ is approximately constant at the beginning of the simulation, so that the hypothesis of homogeneous flow in a Green volume element (taking into account the porosity) well represents the overall behavior of the cell with a von Mises matrix. However, with $C=0, \delta_{0}$ does not depend anymore on the stress state, while still keeping the sudden drop of $\delta_{C}$ necessary for the determination of the coalescence onset.

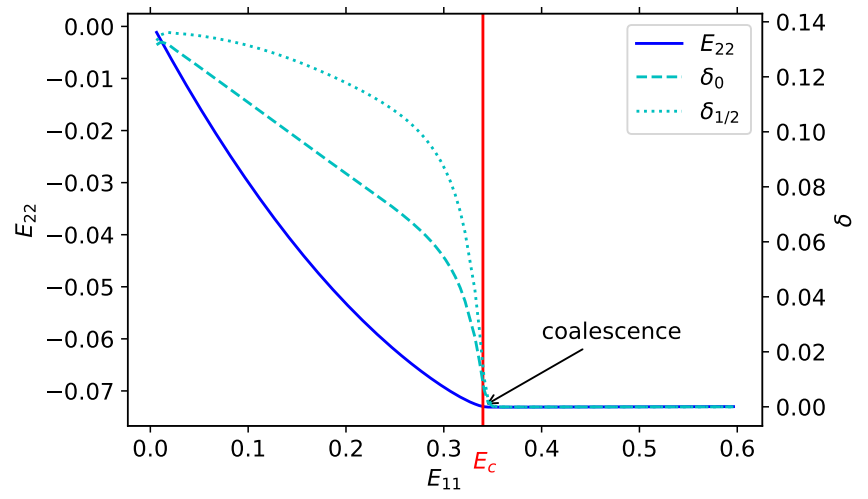

Fig. 15: Detection of failure through simple extension criterion (stabilization of the transverse strain) or vanishing of $\delta$ function. Microstructure $R l$ under the loading condition $T=1, L=-1$ 
A.2 Sensibility analysis regarding the threshold coefficients

Finally we verify that the $\delta$ indicator is a reliable indicator of failure by assessing its sensitivity to the choice of the empirically chosen threshold values. As the equation (16) shows, the determination of the onset of coalescence relies on two thresholds: a relative one $A$, which compares the current value of $\delta$ to its maximum, and an absolute one $B$ mostly active in shear-like conditions. The values for those were chosen as $A=0.05$ and $B=0.005$ but a robust indicator should not be too sensitive to these values.

Figure 16 compares the effect of different $A$ and $B$ values on the $E_{c}-L$ curve (common triaxiality $T=1$, microstructure $R 1$ ). At constant $B$, the effect of $A$ is only visible in the HLEMZ and the LLEMZ, and generally negligible. At constant $A, B$ only affects the coalescence strain values in the SMZ. Although a change in $B$ can modify the strain by 0.05 , the global aspect of the curve is preserved. The determination of failure by the indicator therefore appears robust with respect to changes in the coefficients.

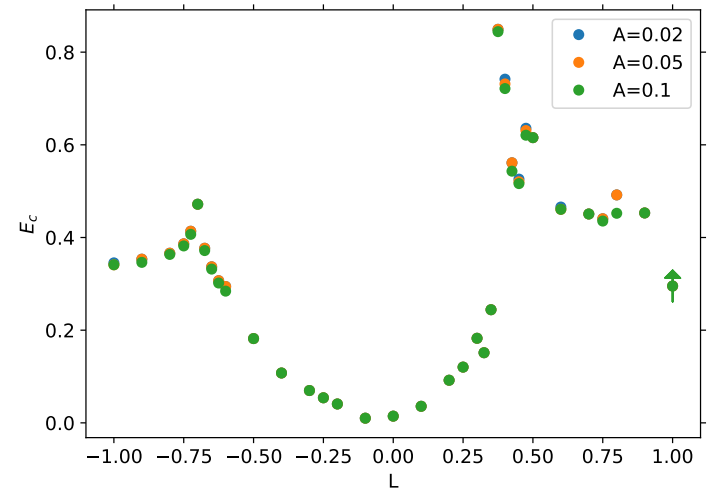

(a) Different values of $A ; B=0.005$

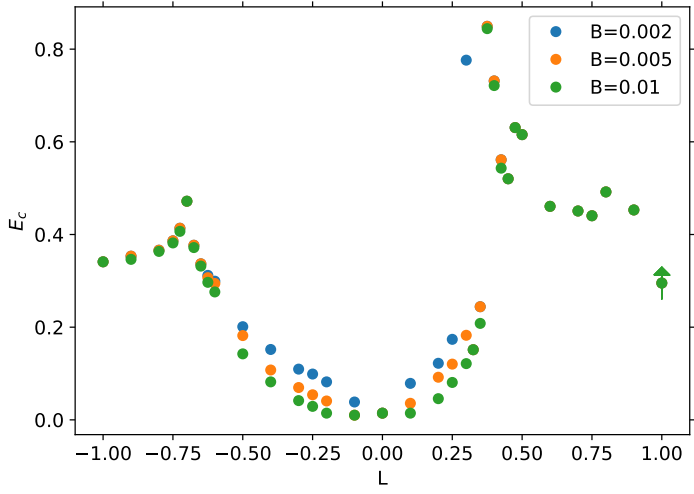

(b) Different values of $B ; A=0.05$

Fig. 16: Effect of varying threshold conditions for the failure indicator on the $E_{c}-L$ curve. All simulations on the $R 1$ microstructure, at $T=1$.

\section{B Effect of different meshing parameters and boundary conditions}

In this section, we review the simulation hypotheses and assess their influence on the results presented up to now, showing therefore how representative the results are and how far they can be generalized. First we verify that finite element discretization effects can be neglected, and investigate the effect of different boundary conditions.

\section{B.1 Effect of the meshing parameters}

All the simulations described up to now were carried out on meshes of cells with the same meshing parameter. To determine the influence of mesh size on coalescence results, the same microstructure $R 1$ was meshed with different meshing parameters $h_{\text {cell }} / r_{0} \in\{1.25,1,0.875,0.625\}$ (with the notation of section 2.1). The maximum element size near the voids is also adapted to keep the ratio $h_{\text {cell }} / h_{\text {void }}=5$ constant. The same loading condition $T=1, L=-1$ is applied to the four meshes. Figure 17 shows that 
stress values during the simulations differ between the meshes, but the relative difference between the finest and coarsest meshes is about $5 \%$, which remains acceptable. The onset of coalescence $E_{c}$ which is our main quantity of interest, is almost identical between the meshes, at $E_{c}=0.33 \pm 1 \%$. Therefore the influence of mesh refinement for random microstructure cells appear limited (although there was only a ratio of 2 between the element sizes of the coarsest and the finest mesh), which justifies the value $h_{\text {cell }}=0.08$ adopted throughout this study.

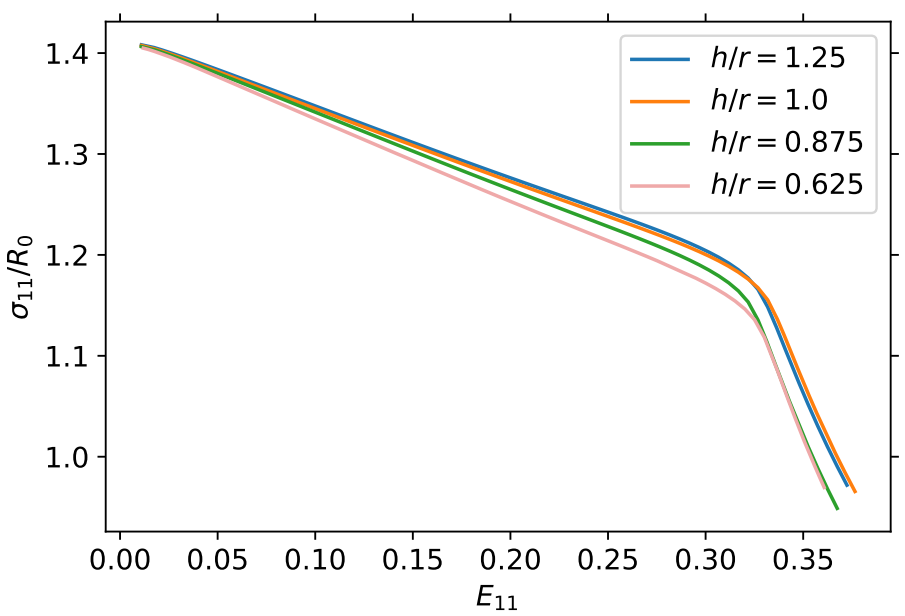

Fig. 17: Cauchy stress during the simulation for several meshes of the $R 1$ random microstructure with different meshing parameters. Loading condition: $T=1, L=-1$

\section{B.2 Effect of the boundary conditions}

We here investigate the influence of boundary conditions. The results from section 4 are first compared to those obtained with different boundary conditions. Namely we investigate the influence of conditions on the average gradient, and of planar faces conditions. The consistency of results at $L=-1$ is also checked by a comparison with simulations on axisymmetric cells.

The conditions imposed on the average gradient $\underset{\sim}{F}$ to prevent rigid body motion are first investigated. In section 2.3 we imposed $\bar{\sim}$ symmetric, as for Ling et al. (2016). However another reasonable choice would be to fix some degrees of freedom at the vertices of the cubic cell, as depicted in figure 18, which is the standard method for boundary value problems. A vertex is already fixed in order to prevent translations, but by fixing two degree of freedom on a second one, and a last one on a third vertex, all rotations are fixed. This can be reformulated as:

$$
\bar{F}_{12}=\bar{F}_{13}=\bar{F}_{23}=0
$$

i.e. $\underset{\sim}{\vec{F}}$ is an upper triangular matrix. Due to the mixed conditions imposed by the macroscopic spring element, the results from the symmetric $\underset{\sim}{\vec{F}}$ case cannot be easily transposed to the triangular $\underset{\sim}{\bar{F}}$ case. These two choices lead to distinct proportional loading path classes and should therefore be compared. 


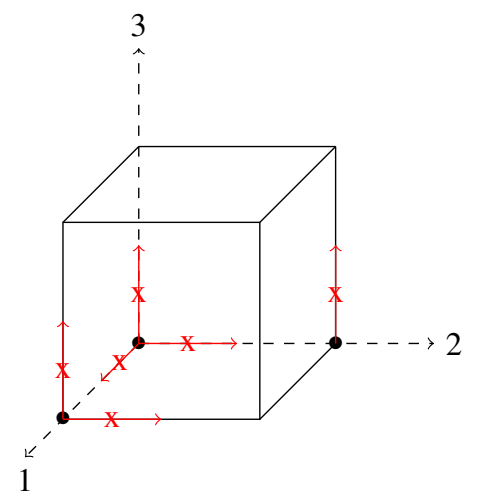

Fig. 18: Conditions on average deformation gradient obtained by fixing some degrees of freedom on vertices of the cubic cell.

On the microstructure $R 1$, at fixed triaxiality $T=1$, simulations were performed for several Lode parameters to compare the two sets of conditions on $\underset{\sim}{\bar{F}}$ (figure 19a). The evolution of $E_{c}$ is close between the two types of conditions, and the same ductility zones can be identified for the triangular gradient condition. However, in that case, cusps seem to be less pronounced than for a symmetric gradient; this may be due to the different treatment of shear components by the two conditions. Therefore the influence of the conditions on $\underset{\sim}{\bar{F}}$ remains limited.

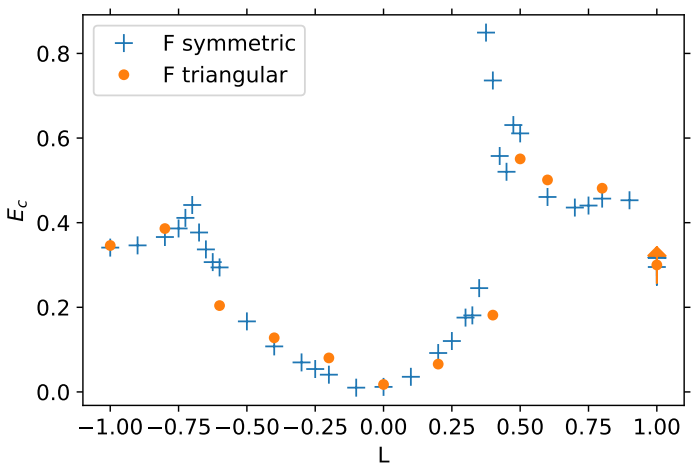

(a) Conditions on the average deformation gradient. (Computations on the microstructure R1)

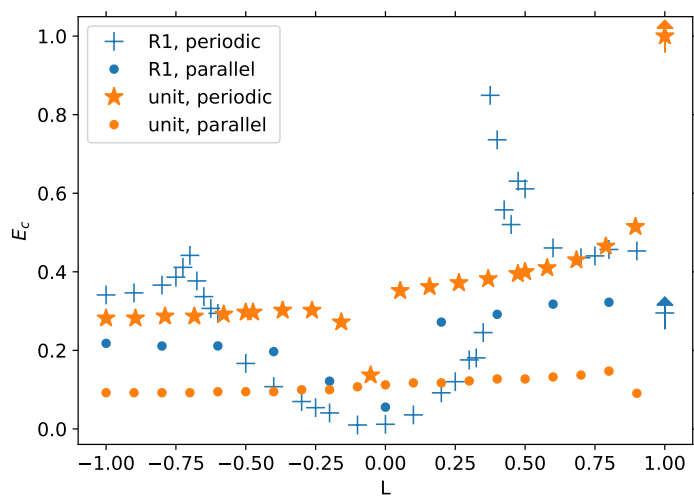

(b) Periodic and parallel faces boundary conditions

Fig. 19: Influence of boundary conditions on the response of the cell. All computations at triaxiality $T=1$.

We then compare the effects of periodic and parallel faces boundary conditions. Parallel faces conditions mean that the cubic cell retains parallel flat faces throughout the computation (for instance all the points on the $x_{0}=0$ face have the same $x$-displacement). This condition is more constraining than periodic boundary conditions. As the comparison in figure 19b shows, the two types of conditions lead to qualitatively different responses. For the parallel faces, no separation between three ductility zones can be seen (except near $L=0$ ) and the response of the random microstructure is closer to that typical of the unit cell. Moreover no decrease of ductility near $L=0$ is observed for the unit cell. Results for the unit cell differ between the parallel faces and periodic 
boundary conditions, because in the periodic case, faces are allowed not to remain strictly parallel and planar. On the contrary, boundary conditions made of parallel sides strongly hinder the shear mode failure and only the extension mode remains possible. The competition between these two modes tends to postpone failure (see the cusps on figure 4). Therefore, the reduced competition between modes may explain an earlier coalescence for parallel unit cells. The preceding results show that boundary conditions exert a strong influence on the response of the cell.

Finally, the consistency of results obtained at $T=1$ is checked. As this type of loading is axisymmetric, a computation with a $2 \mathrm{D}$ axisymmetric unit cell was also performed for comparison. Such unit cells are frequent in ductile fracture studies (Morin, Leblond, and Benzerga (2015) for instance). The diameter and the height of the cylinder were chosen equal to $L_{c u b e}$. The porosity is still $6 \%$, so the radius of the void was modified to $0.22 L_{\text {cube }}$. The boundary conditions for this cell differ slightly from those described in section 2.3: they are no more periodic and are replaced by straight edges conditions. Besides the virtual constant triaxiality element is not linked to the average deformation gradient but to the displacement of the top left node.

Figure 20 compare results for the unit cell and the $2 \mathrm{D}$ axisymmetric cell at varying stress triaxiality for $L=-1$. In this type of loading, the unit cell was shown in section 4 to exhibit the same behavior as random microstructures, compatible with a Rice-Tracey evolution. At initial porosity $f_{0}=6 \%$, the axisymmetric cell presents however a significantly higher exponent (in absolute value) for the evolution of $E_{c}$ with respect to $T$. This effect seems due to the relatively high porosity in the axisymmetric cell: as depicted in figure 20, the evolution of $E_{c}$ with respect to $T$ for the low porosity $f_{0}=1 \%$ axisymmetric cell is much closer to the one predicted by Rice and Tracey.

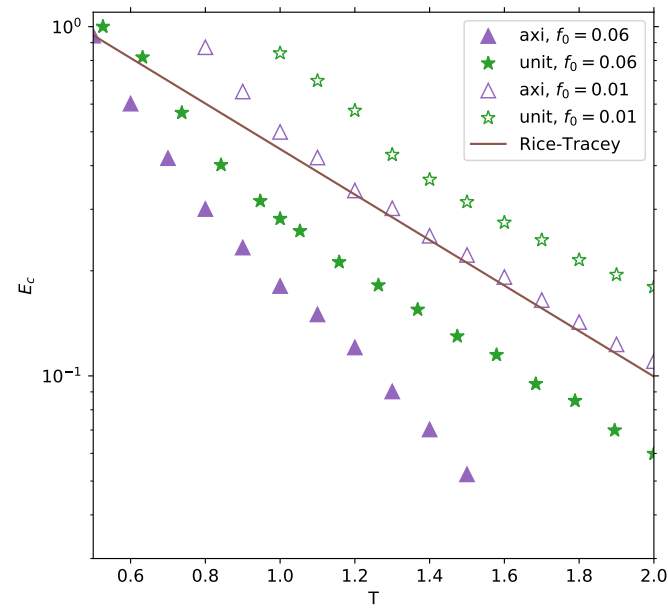

Fig. 20: Evolution of the strain at coalescence with respect to $T$ for the cubic and 2D axisymmetric unit cells for porosity values $f_{0}=6 \%$ and $1 \%$ (constant Lode parameter $L=-1$ ). 


\section{References}

Bandstra, JP and Koss, DA (2008). "On the influence of void clusters on void growth and coalescence during ductile fracture". Acta Materialia 56: 4429-4439. DoI: $10.1016 /$ j . actamat.2008.05.009.

Bao, Y and Wierzbicki, T (2004). "On fracture locus in the equivalent strain and stress triaxiality space". International Journal of Mechanical Sciences 46: 81-98. DOI: $10.1016 /$ j . i jmecsci. 2004.02. 006.

Barsoum, I and Faleskog, J (2007). "Rupture mechanisms in combined tension and shear-Micromechanics". International Journal of Solids and Structures 44: 5481-5498. DOI: 10.1016 / j . i j sol str.2007.01. 010.

Barsoum, I and Faleskog, J (2011). "Micromechanical analysis on the influence of the Lode parameter on void growth and coalescence”. International Journal of Solids and Structures 48: 925-938. DoI: $10.1016 / \mathrm{j}$. ijsolstr.2010.11.028.

Becker, R (1987). "The effect of porosity distribution on ductile failure”. Journal of the Mechanics and Physics of Solids 35: 577-599. DOI: $10.1016 / 0022-5096$ (87) $90018-4$.

Benzerga, AA and Leblond, JB (2014). "Effective Yield Criterion Accounting for Microvoid Coalescence". Journal of Applied Mechanics 81: 031009. DOI: $10.1115 / 1.4024908$.

Besson, J, ed. (2004). Local Approach to Fracture. Paris: Les Presses de l'École des Mines.

Besson, J (2010). "Continuum models of ductile fracture : A review”. International Journal of Damage Mechanics 19: 3-52. DOI: 10.1177/1056789509103482.

Besson, J, Cailletaud, G, Chaboche, JL, Forest, S, and Blétry, M (2009). Non-Linear Mechanics of Materials. Solid Mechanics and its Applications. Springer.

Besson, J and Foerch, R (1998). “Object-Oriented Programming Applied to the Finite Element Method Part I. General Concepts”. Revue Européenne des Éléments Finis 7. Publisher: Taylor \& Francis _eprint: https://doi.org/10.1080/12506559.1998.10511321: 535-566. DOI: 10 . $1080 / 12506559$. 1998 . 10511321.

Bilger, N, Auslender, F, Bornert, M, Michel, JC, Moulinec, H, Suquet, P, and Zaoui, A (2005). "Effect of a nonuniform distribution of voids on the plastic response of voided materials: a computational and statistical analysis". International Journal of Solids and Structures 42: 517-538. DOI: 10.1016 / j. i jsolstr. 2004.06 .048$. 
Bilger, N, Auslender, F, Bornert, M, Moulinec, H, and Zaoui, A (2007). "Bounds and estimates for the effective yield surface of porous media with a uniform or a nonuniform distribution of voids". European Journal of Mechanics - A/Solids 26: 810-836. DoI: 10.1016/j.euromechso1.2007.01.004.

Boyce, BL, Kramer, SLB, Bosiljevac, TR, et al. (2016). "The second Sandia Fracture Challenge: predictions of ductile failure under quasi-static and moderate-rate dynamic loading”. International Journal of Fracture 198: 5-100. DOI: $10.1007 / \mathrm{s} 10704-016-0089-7$.

Boyce, BL, Kramer, SLB, Fang, HE, et al. (2014). "The Sandia Fracture Challenge: blind round robin predictions of ductile tearing". International Journal of Fracture 186: 5-68. DOI: 10.1007 / s10704-013-9904-6. Brown, L and Embury, J (1973). "Initiation and growth of voids at second phase particles". Inst Met (London) Monogr Rep Ser 1: 164-169.

Brown, MB and Forsythe, AB (1974). "Robust Tests for the Equality of Variances". Journal of the American Statistical Association 69: 364-367. DOI: $10.1080 / 01621459.1974 .10482955$.

Cawthorne, C and Fulton, EJ (1967). "Voids in Irradiated Stainless Steel". Nature 216. Number: 5115 Publisher: Nature Publishing Group: 575-576. DOI: 10 . 1038/216575a0.

Coenen, EWC, Kouznetsova, VG, and Geers, MGD (2012). "Novel boundary conditions for strain localization analyses in microstructural volume elements". International Journal for Numerical Methods in Engineering 90._eprint: https://onlinelibrary.wiley.com/doi/pdf/10.1002/nme.3298: 1-21. DOI: 10 . 1002 /nme. 3298. Dæhli, LEB, Morin, D, Børvik, T, Benallal, A, and Hopperstad, OS (2020). “A Numerical Study on Ductile Failure of Porous Ductile Solids With Rate-Dependent Matrix Behavior”. Journal of Applied Mechanics 87: 031014. DOI: $10.1115 / 1.4045524$.

Danas, K and Ponte Castañeda, P (2009). “A finite-strain model for anisotropic viscoplastic porous media: I - Theory". European Journal of Mechanics - A/Solids 28: 387-401. DOI: 10.1016 / j . euromechsol . 2008.11 .002 .

Danas, K and Ponte Castañeda, P (2012). "Influence of the Lode parameter and the stress triaxiality on the failure of elasto-plastic porous materials". International Journal of Solids and Structures 49: 1325-1342. DOI: $10.1016 / j . i j s o l s t r .2012 .02 .006$.

Dunand, M and Mohr, D (2011). "On the predictive capabilities of the shear modified Gurson and the modified Mohr-Coulomb fracture models over a wide range of stress triaxialities and Lode angles". Journal of the Mechanics and Physics of Solids 59: 1374-1394. DoI: 10.1016/j . jmps.2011.04.006. 
Dunand, M and Mohr, D (2014). "Effect of Lode parameter on plastic flow localization after proportional loading at low stress triaxialities". Journal of the Mechanics and Physics of Solids 66: 133-153. DOI: $10.1016 / j . j m p s .2014 .01 .008$.

Fritzen, F, Forest, S, Böhlke, T, Kondo, D, and Kanit, T (2012). "Computational homogenization of elasto-plastic porous metals". International Journal of Plasticity 29: 102-119. DOI: $10.1016 / \mathrm{j}$. i jplas .2011.08. 005.

Fritzen, F, Forest, S, Kondo, D, and Böhlke, T (2013). "Computational homogenization of porous materials of Green type". Computational Mechanics 52: 121-134. DOI: 10.1007 /s $00466-012-0801-z$.

Gamito, M and Maddock, S (2009). “Accurate Multidimensional Poisson-Disk Sampling”. ACM Trans. Graph. 29: 8. DOI: $10.1145 / 1640443.1640451$.

Gao, X, Zhang, T, Hayden, M, and Roe, C (2009). "Effects of the stress state on plasticity and ductile failure of an aluminum 5083 alloy”. International Journal of Plasticity 25: 2366-2382. DOI: 10.1016 / j. i jplas . 2009.03 .006$.

Gilioli, A, Manes, A, Giglio, M, and Allahverdizadeh, N (2013). "Effect of Triaxiality and Lode Angle on the Plasticity Behaviour of a Ti-6Al-4V Titanium Alloy”. Key Engineering Materials 577-578: 413-416. DOI: https://doi.org/10.4028/www.scientific.net/KEM.577-578.413.

Gologanu, M, Leblond, JB, and Devaux, J (2001). "Theoretical models for void coalescence in porous ductile solids. II. Coalescence in columns". International Journal of Solids and Structures 38: 5595-5604. DOI: $10.1016 / \mathrm{S} 0020-7683(00) 00355-3$.

Green, RJ (1972). “A plasticity theory for porous solids”. International Journal of Mechanical Sciences 14: 215-224. DOI: $10.1016 / 0020-7403(72) 90063-\mathrm{X}$.

Guo, HJ, Ling, C, Busso, EP, Zhong, Z, and Li, DF (2020). "Crystal plasticity based investigation of micro-void evolution under multi-axial loading conditions". International Journal of Plasticity 129: 102673. DOI: $10.1016 / j . i j p l a s .2020 .102673$.

Guo, TF and Wong, WH (2018). "Void-sheet analysis on macroscopic strain localization and void coalescence". Journal of the Mechanics and Physics of Solids 118: 172-203. DOI: $10.1016 / j \cdot j m p s .2018 .05 .002$. Gurson, AL (1977). "Continuum Theory of Ductile Rupture by Void Nucleation and Growth: Part I-Yield Criteria and Flow Rules for Porous Ductile Media”. Journal of Engineering Materials and Technology 99: 2-15. DOI: $10.1115 / 1.3443401$.

Heiman, GW (2001). Understanding research methods and statistics: An integrated introduction for psychology. Boston: Houghton, Mifflin and Company. 
Helbert, AL, Feaugas, X, and Clavel, M (1996). "The influence of stress triaxiality on the damage mechanisms in an equiaxed Ti-6AI-4V alloy”. Metallurgical and Materials Transactions A 27: 3043-3058. DOI: 10 . $1007 / \mathrm{BF} 02663853$.

Hure, J (2021). "Yield criterion and finite strain behavior of random porous isotropic materials". European Journal of Mechanics - A/Solids 85: 104143. DoI: 10.1016/j.euromechsol .2020.104143.

Hutchinson, JW and Tvergaard, V (2012). "Comment on "Influence of the Lode parameter and the stress triaxiality on the failure of elasto-plastic porous materials" by K. Danas and P. Ponte Castañeda". International Journal of Solids and Structures 49: 3484-3485. DoI: $10.1016 /$ j. i jsolstr.2012.07.009.

Kanit, T, Forest, S, Galliet, I, Mounoury, V, and Jeulin, D (2003). "Determination of the size of the representative volume element for random composites: statistical and numerical approach". International Journal of Solids and Structures 40: 3647-3679. DOI: $10.1016 /$ s $0020-7683$ ( 03 ) 00143-4.

Keralavarma, SM, Reddi, D, and Benzerga, AA (2020). "Ductile failure as a constitutive instability in porous plastic solids". Journal of the Mechanics and Physics of Solids 139: 103917. DOI: $10.1016 /$ j . jmps . 2020.103917.

Khan, IA and Bhasin, V (2017). "On the role of secondary voids and their distribution in the mechanism of void growth and coalescence in porous plastic solids". International Journal of Solids and Structures 108: 203-215. DOI: 10.1016/j.ijsolstr.2016.12.016.

Khdir, YK, Kanit, T, Zaïri, F, and Naït-Abdelaziz, M (2014). "Computational homogenization of plastic porous media with two populations of voids”. Materials Science and Engineering: A 597: 324-330. DOI: $10.1016 / j . m s e a .2013 .12 .095$.

Khdir, YK, Kanit, T, Zaïri, F, and Naït-Abdelaziz, M (2015). “A computational homogenization of random porous media: Effect of void shape and void content on the overall yield surface”. European Journal of Mechanics - A/Solids 49: 137-145. DoI: $10.1016 / j$.euromechsol.2014.07.001.

Koplik, J and Needleman, A (1988). "Void growth and coalescence in porous plastic solids". International Journal of Solids and Structures 24: 835-853. DOI: $10.1016 / 0020-7683$ (88) 90051-0.

Kramer, SLB et al. (2019). "The third Sandia fracture challenge: predictions of ductile fracture in additively manufactured metal". International Journal of Fracture 218: 5-61. DOI: 10.1007 / s10704-019$00361-1$

Lacourt, L (2019). "Étude numérique de la nocivité des défauts". Thèse de doctorat. Paris: Paris Sciences \& Lettres. 
Lacourt, L, Ryckelynck, D, Forest, S, Rancourt, Vd, and Flouriot, S (2020). "Hyper-reduced direct numerical simulation of voids in welded joints via image-based modeling”. International Journal for Numerical Methods in Engineering 121: 2581-2599. DOI: $10.1002 / \mathrm{nme} .6320$.

Leblond, JB and Mottet, G (2008). "A theoretical approach of strain localization within thin planar bands in porous ductile materials”. Comptes Rendus Mécanique 336: 176-189. DoI: 10.1016 / j . crme.2007. 11.008.

Li, XY, Hao, Q, Shi, YW, Lei, YP, and Marquis, G (2003). "Influence of mechanical mismatching on the failure of welded joints by void nucleation and coalescence". International Journal of Pressure Vessels and Piping 80: 647-654. DOI: $10.1016 / \mathrm{S} 0308-0161$ ( 03 ) $00094-2$.

Ling, C, Besson, J, Forest, S, Tanguy, B, Latourte, F, and Bosso, E (2016). “An elastoviscoplastic model for porous single crystals at finite strains and its assessment based on unit cell simulations". International Journal of Plasticity 84: 58-87. DOI: $10.1016 / j . i j p l a s .2016 .05 .001$.

Liu, ZG, Wong, WH, and Guo, TF (2016). "Void behaviors from low to high triaxialities: Transition from void collapse to void coalescence". International Journal of Plasticity 84: 183-202. DOI: $10.1016 / j$. ijplas.2016.05.008.

Luo, T and Gao, X (2018). "On the prediction of ductile fracture by void coalescence and strain localization". Journal of the Mechanics and Physics of Solids 113: 82-104. DOI: $10.1016 / j$.jmps . 2018.02 .002$.

Matern, B (1986). Spatial Variation. 2nd ed. Lecture Notes in Statistics. New York: Springer-Verlag. DOI: $10.1007 / 978-1-4615-7892-5$.

McClintock, FA (1968). "A Criterion for Ductile Fracture by the Growth of Holes”. Journal of Applied Mechanics 35. Publisher: American Society of Mechanical Engineers Digital Collection: 363-371. DOI: $10.1115 / 1.3601204$.

Morin, D, Blystad Dæhli, LE, Børvik, T, Benallal, A, and Hopperstad, OS (2019). "Numerical study of ductile failure under non-proportional loading”. European Journal of Mechanics - A/Solids 74: 221-241. DOI: $10.1016 / j$.euromechsol.2018.11.001.

Morin, L, Leblond, JB, and Benzerga, AA (2015). "Coalescence of voids by internal necking: Theoretical estimates and numerical results". Journal of the Mechanics and Physics of Solids 75: 140-158. DOI $10.1016 / j \cdot j m p s .2014 .11 .009$.

Morin, L, Leblond, JB, Benzerga, AA, and Kondo, D (2016). "A unified criterion for the growth and coalescence of microvoids". Journal of the Mechanics and Physics of Solids 97: 19-36. DOI: $10.1016 / j$. jmps . 2016.01 .013$. 
Needleman, A and Tvergaard, V (1992). "Analyses of plastic flow localization in metals". Applied Mechanics Reviews 45: 3-18. DOI: 10.1115/1.3121390.

Nguyen, VD, Pardoen, T, and Noels, L (2020). "A nonlocal approach of ductile failure incorporating void growth, internal necking, and shear dominated coalescence mechanisms". Journal of the Mechanics and Physics of Solids 137: 103891. DOI: $10.1016 / j \cdot j m p s .2020 .103891$.

Pedregosa, F et al. (2011). "Scikit-learn: Machine Learning in Python". Journal of Machine Learning Research 12: $2825-2830$.

Ponte Castañeda, P and Willis, JR (1995). "The effect of spatial distribution on the effective behavior of composite materials and cracked media". Journal of the Mechanics and Physics of Solids 43: 1919-1951. DOI: $10.1016 / 0022-5096(95) 00058-Q$.

Rice, JR and Tracey, DM (1969). "On the ductile enlargement of voids in triaxial stress fields". Journal of the Mechanics and Physics of Solids 17: 201-217.

Rice, JR (1976). "The localization of deformation". In: Theoretical and Applied Mechanics, Proceedings of the 14th IUTAM congress, Delft. Ed. by WT Koiter. Delft: North-Holland Publishing Company: 207-220.

Sarre, B (2018). "Influence du soudage laser Nd:YAG sur les propriétés métallurgiques et mécaniques de l'alliage de titane TA6V". Thèse de doctorat. Troyes: Université de Technologie de Troyes.

Schöberl, J (1997). "NETGEN - An advancing front 2D/3D-mesh generator based on abstract rules". Computing and Visualization in Science 1: 41-52.

Shakoor, M, Bernacki, M, and Bouchard, PO (2015). "A new body-fitted immersed volume method for the modeling of ductile fracture at the microscale: Analysis of void clusters and stress state effects on coalescence”. Engineering Fracture Mechanics 147: 398-417. DOI: 10.1016 / j .eng fracmech. 2015. 06.057.

Shakoor, M, Bernacki, M, and Bouchard, PO (2018). "Ductile fracture of a metal matrix composite studied using 3D numerical modeling of void nucleation and coalescence". Engineering Fracture Mechanics 189: 110-132. DoI: $10.1016 / j$.engfracmech.2017.10.027.

Tekoğlu, C, Hutchinson, JW, and Pardoen, T (2015). "On localization and void coalescence as a precursor to ductile fracture". Philosophical Transactions of the Royal Society A: Mathematical, Physical and Engineering Sciences 373: 20140121. DOI: 10.1098/rsta.2014.0121.

Thomason, PF (1985). "Three-dimensional models for the plastic limit-loads at incipient failure of the intervoid matrix in ductile porous solids”. Acta Metallurgica 33: 1079-1085. DOI: $10.1016 / 0001-6160$ (85) $90201-9$. 
Torki, ME (2019). "A unified criterion for void growth and coalescence under combined tension and shear". International Journal of Plasticity 119: 57-84. DOI: $10.1016 / j . i j p l a s .2019 .02 .002$.

Trejo Navas, VM, Bernacki, M, and Bouchard, PO (2018). "Void growth and coalescence in a three-dimensional non-periodic void cluster". International Journal of Solids and Structures 139-140: 65-78. DOI: $10.1016 /$ j.ijsolstr.2018.01.024

Tvergaard, V (2016). "Effect of void cluster on ductile failure evolution". Meccanica 51: 3097-3105. DOI: $10.1007 / \mathrm{s} 11012-016-0537-5$

Tvergaard, V (2017). "Nucleation from a cluster of inclusions, leading to void coalescense". International Journal of Mechanical Sciences 133: 631-638. DoI: 10.1016/j.i jmecsci.2017.09.027.

Tvergaard, V and Needleman, A (1984). "Analysis of the cup-cone fracture in a round tensile bar". Acta Metallurgica 32: 157-169. DoI: https://doi .org/10.1016/0001-6160 (84) 90213-X.

Vincent, PG, Monerie, Y, and Suquet, P (2009). "Porous materials with two populations of voids under internal pressure: I. Instantaneous constitutive relations". International Journal of Solids and Structures 46: 480-506. DOI: $10.1016 / j . i j s o l s t r .2008 .09 .003$.

Virtanen, P et al. (2020). “SciPy 1.0: fundamental algorithms for scientific computing in Python”. Nature Methods 17. Number: 3 Publisher: Nature Publishing Group: 261-272. DOI: 10.1038 /s 41592-019-0686-2. Vishwakarma, V and Keralavarma, SM (2019). "Micromechanical modeling and simulation of the loading path dependence of ductile failure by void growth to coalescence". International Journal of Solids and Structures 166: 135-153. DOI: 10.1016/j.ijsolstr.2019.02.015.

Wong, WH and Guo, TF (2015). “On the energetics of tensile and shear void coalescences”. Journal of the Mechanics and Physics of Solids 82: 259-286. DOI: $10.1016 / j \cdot j m p s .2015 .05 .013$.

www.zset-software.com (2020).

Xiao, X, Mu, Z, Pan, H, and Lou, Y (2018). "Effect of the Lode parameter in predicting shear cracking of 2024-T351 aluminum alloy Taylor rods". International Journal of Impact Engineering 120: 185-201. DOI: $10.1016 / j . i j i m p e n g .2018 .06 .008$

Zhai, J, Luo, T, Gao, X, Graham, SM, Baral, M, Korkolis, YP, and Knudsen, E (2016). "Modeling the ductile damage process in commercially pure titanium”. International Journal of Solids and Structures 91: 26-45. DOI: $10.1016 / j . i j s o l s t r .2016 .04 .031$

Zhang, KS, Bai, JB, and François, D (1999). "Ductile fracture of materials with high void volume fraction". International Journal of Solids and Structures 36: 3407-3425. DOI: 10 . 1016 / S0020-7683 (98) $00157-7$. 
Zhang, K, Badreddine, H, and Saanouni, K (2020). "Ductile fracture prediction using enhanced CDM model with Lode angle-dependency for titanium alloy Ti-6Al-4V at room temperature". Journal of Materials Processing Technology 277: 116462. DoI: $10.1016 / j$.jmatprotec.2019.116462.

Zhu, JC, Ben Bettaieb, M, and Abed-Meraim, F (2020). "Investigation of the competition between void coalescence and macroscopic strain localization using the periodic homogenization multiscale scheme". Journal of the Mechanics and Physics of Solids 143: 104042. DOI: $10.1016 /$ j. jmps . 2020.104042. Zhu, Y, Engelhardt, MD, and Kiran, R (2018). "Combined effects of triaxiality, Lode parameter and shear stress on void growth and coalescence”. Engineering Fracture Mechanics 199: 410-437. DOI: $10.1016 / j$. engfracmech.2018.06.008. 